\title{
Adiabatic limit, Bismut-Freed connection, and the real analytic torsion form
}

\author{
Xianzhe Dai and Weiping Zhang
}

\begin{abstract}
For a complex flat vector bundle over a fibered manifold, we consider the 1-parameter family of certain deformed sub-signature operators introduced by Ma-Zhang in MZ. We compute the adiabatic limit of the Bismut-Freed connection associated to this family and show that the Bismut-Lott analytic torsion form shows up naturally under this procedure.
\end{abstract}

\section{Introduction}

Adiabatic limit refers to the geometric degeneration when metric in certain directions are blown up, while the remaining directions are kept fixed.

Typically, the underlying manifold has a so called fibration structure (or fiber bundle structure). That is

$$
F \longrightarrow M \stackrel{\pi}{\longrightarrow} B
$$

where $\pi$ is a submersion and $F \simeq F_{b}=\pi^{-1}(b)$, for $b \in B$. Given a submersion metric on $M$ :

$$
g=\pi^{*} g_{B}+g_{F},
$$

the adiabatic limit refers to the limit as $\epsilon \rightarrow 0$ of

$$
g_{\epsilon}=\epsilon^{-2} \pi^{*} g_{B}+g_{F} .
$$

This is first introduced by Witten $[\mathrm{W}]$ in his famous work on global gravitational anomalies.

Witten considered the adiabatic limit of the eta invariant of Atiyah-PatodiSinger APS1]- APS3]. Full mathematical treatment and generalizations are given by Bismut-Freed [BF], Cheeger [C], Bismut-Cheeger [BC1], Dai [D] among others. The adiabatic limit of the eta invariant gives rise to the Bismut-Cheeger eta form, a canonically defined differential form on the base $B$. The eta form is a higher dimensional generalization of the eta invariant as it gives the boundary contribution of the family index theorem for manifolds with boundaries, see Bismut-Cheeger [BC2, BC3], and Melrose-Piazza [MP1, MP2]. The degree zero component of the eta form here is exactly the eta invariants of the fibers. The nonzero degree components therefore contains new geometric information about the fibration. 
Another important geometric invariant is the analytic torsion. The adiabatic limit of the analytic torsion has been considered by Dai-Melrose DM] (see also the topological treatment of Fried [Fri], Freed [Fr], and Lück-SchickThielmann [LST]). In contrast to the case of the eta invariant, the adiabatic limit here does not give rise to a higher invariant. This is because the associated characteristic class involved here is the Pfaffian, a top form which kills any possible higher degree components arising from the adiabatic limit.

It should be noted that there is a complex analogue of the analytic torsion for complex manifolds called the holomorphic torsion. Its adiabatic limit has been considered by Berthomieu-Bismut $[\mathrm{BerB}$. And it does produce the holomorphic torsion form of Bismut-Köhler [BK]. The difference can be explained by the fact that the characteristic class here is the Todd class - a stable class.

There is another way to view the higher invariants, namely via transgression. The eta form transgresses between the Chern-Weil representative of the family index and its Atiyah-Singer representative. Similarly, the holomorphic torsion form is the double transgression of the family index in the complex setting. Bismut-Lott $[\mathrm{BL}$ ] uses this view point to define the real analytic torsion form, a higher dimensional generalization of the analytic torsion. It is a canonical transgression of certain odd cohomology classes.

There remains the question of whether the real analytic torsion form can be obtain from the adiabatic limit process. The purpose of this paper is to answer this question in the affirmative. We show that, if one considers the BismutFreed connection of the 1-parameter family of certain deformed sub-signature operators introduced by Ma-Zhang in [MZ], its adiabatic limit essentially gives rise to the Bismut-Lott real analytic torsion form. In fact, it is precisely the positive degree component of the real analytic torsion form that is captured here. This should be compared with $[\mathrm{DM}]$ where the adiabatic limit of the analytic torsion captures only the degree 0 part of the real analytic torsion form.

The paper is organized as follows. We first look at the finite dimensional case in Section 2. Thus in 2.1, we introduce flat cochain complexes, flat superconnections and their rescalings. The family of deformed sub-signature operators is then introduced in 92.2 . After some preparatory results, we define an invariant which should be interpreted as the imaginary part of the Bismut-Freed connection form for the family of the deformed sub-signature operators. Finally in 92.3 , we study the adiabatic limit of our invariant. The fibration case is set up as an infinite dimensional analog and studied in Section 3. The flat superconnection in this case is the Bismut-Lott superconnection and is recalled in $\S 3.1$. In $§ 3.2$, we discuss the analog of the deformed sub-signature operators in the fibration case. Then we look into the Bismut-Freed connection and define a corresponding invariant in $\S 3.3$. Finally, we study the adiabatic limit of our invariant in $\S 3.4$. Our main result is stated in Theorem 3.8, In $\S 3.5$, we compare the Bismut-Lott real analytic torsion form with the torsion form coming out of the adiabatic limit. 


\section{The finite dimensional case}

In this section, we study the finite dimensional case where instead of a flat vector bundle over a fibered manifold, we consider the situation of a flat cochain complex over an even dimensional manifold. This fits well with the structures considered in $[\mathrm{BL}]$ and $[\mathrm{MZ}$. The fibered manifold case is the infinite dimensional analog which will be considered in the next section.

\subsection{Supperconnections and flat cochain complex}

Let $(E, v)$ be a $\mathbb{Z}$-graded cochain complex of finite rank complex vector bundles over a closed manifold $B$,

$$
(E, v): \quad 0 \rightarrow E^{0} \stackrel{v}{\rightarrow} E^{1} \stackrel{v}{\rightarrow} \cdots \stackrel{v}{\rightarrow} E^{n} \rightarrow 0 .
$$

Let $\nabla^{E}=\oplus_{i=0}^{n} \nabla^{E^{i}}$ be a $\mathbb{Z}$-graded connection on $E$. We call $\left(E, v, \nabla^{E}\right)$ a flat cochain complex if the following two conditions hold,

$$
\left(\nabla^{E}\right)^{2}=0, \quad\left[\nabla^{E}, v\right]=0 .
$$

Let $h^{E}=\oplus_{i=0}^{n} h^{E^{i}}$ be a $\mathbb{Z}$-graded hermitian metric on $E$ and denote by $v^{*}: E^{*} \rightarrow E^{*-1}$ the adjoint of $v$ with respect to $h^{E}$. Let $\left(\nabla^{E}\right)^{*}$ denote the adjoint connection of $\nabla^{E}$ with respect to $g^{E}$. Then (cf. [BZ, (4.1), (4.2)] and [BL, $\S 1(\mathrm{~g})])$

$$
\left(\nabla^{E}\right)^{*}=\nabla^{E}+\omega\left(E, h^{E}\right)
$$

where

$$
\omega\left(E, h^{E}\right)=\left(h^{E}\right)^{-1}\left(\nabla^{E} h^{E}\right) .
$$

Consider the superconnections on $E$ in the sense of Quillen Q defined by

$$
A^{\prime}=\nabla^{E}+v, \quad A^{\prime \prime}=\left(\nabla^{E}\right)^{*}+v^{*} .
$$

Let $N \in \operatorname{End}(E)$ denote the number operator of $E$ which acts on $E^{i}$ by multiplication by $i$. We extend $N$ to an element of $\Omega^{0}(B, \operatorname{End}(E))$.

Following [BL, (2.26), (2.30)], for any $u>0$, set

$$
\begin{aligned}
& C_{u}^{\prime}=u^{N / 2} A^{\prime} u^{-N / 2}=\nabla^{E}+\sqrt{u} v, \\
& C_{u}^{\prime \prime}=u^{-N / 2} A^{\prime \prime} u^{N / 2}=\left(\nabla^{E}\right)^{*}+\sqrt{u} v^{*}, \\
& C_{u}=\frac{1}{2}\left(C_{u}^{\prime}+C_{u}^{\prime \prime}\right), \quad D_{u}=\frac{1}{2}\left(C_{u}^{\prime \prime}-C_{u}^{\prime}\right) .
\end{aligned}
$$

Then we have

$$
C_{u}^{2}=-D_{u}^{2}, \quad\left[C_{u}, D_{u}\right]=0 .
$$

Let

$$
\nabla^{E, e}=\nabla^{E}+\frac{1}{2} \omega\left(E, h^{E}\right)
$$


be the Hermitian connection on $\left(E, h^{E}\right)(\mathrm{cf}$. [BL, (1.33)] and [BZ, (4.3)]). Then

$$
C_{u}=\nabla^{E, e}+\frac{\sqrt{u}}{2}\left(v+v^{*}\right)
$$

is a superconnection on $E$, while

$$
D_{u}=\frac{1}{2} \omega\left(E, h^{E}\right)+\frac{\sqrt{u}}{2}\left(v^{*}-v\right)
$$

is an odd element in $C^{\infty}\left(B, \Lambda^{*}(B) \widehat{\otimes} \operatorname{End}(E)\right)$.

\subsection{Deformed signature operators and the Bismut-Freed con- nection}

We assume in the rest of this section that $p=\operatorname{dim} B$ is even and $B$ is oriented.

Let $g^{T B}$ be a Riemannian metric on $T B$. For $X \in T B$, let $c(X), \widehat{c}(X)$ be the Clifford actions on $\Lambda\left(T^{*} B\right)$ defined by $c(X)=X^{*}-i_{X}, \widehat{c}(X)=X^{*}+i_{X}$, where $X^{*} \in T^{*} B$ corresponds to $X$ via $g^{T B}$ (cf. [BL, (3.18)] and [BZ, $\left.\S 4(\mathrm{~d})\right]$ ). Then for any $X, Y \in T B$,

$$
\begin{aligned}
& c(X) c(Y)+c(Y) c(X)=-2\langle X, Y\rangle, \\
& \widehat{c}(X) \widehat{c}(Y)+\widehat{c}(Y) \widehat{c}(X)=2\langle X, Y\rangle, \\
& c(X) \widehat{c}(Y)+\widehat{c}(Y) c(X)=0 .
\end{aligned}
$$

Let $e_{1}, \cdots, e_{p}$ be a (local) oriented orthonormal basis of $T B$. Set

$$
\tau=(\sqrt{-1})^{\frac{p(p+1)}{2}} c\left(e_{1}\right) \cdots c\left(e_{p}\right) .
$$

Then $\tau$ is a well-defined self-adjoint element such that

$$
\tau^{2}=\left.\mathrm{Id}\right|_{\Lambda\left(T^{*} B\right)}
$$

Let $\mu$ be a Hermitian vector bundle on $B$ carrying a Hermitian connection $\nabla^{\mu}$ with the curvature denoted by $R^{\mu}=\left(\nabla^{\mu}\right)^{2}$. Let $\nabla^{T B}$ be the Levi-Civita connection on $\left(T B, g^{T B}\right)$ with its curvature $R^{T B}$. Let $\nabla^{\Lambda\left(T^{*} B\right)}$ be the Hermitian connection on $\Lambda\left(T^{*} B\right)$ canonically induced from $\nabla^{T B}$. Let $\nabla^{\Lambda\left(T^{*} B\right) \otimes \mu \otimes E, e}$ be the tensor product connection on $\Lambda\left(T^{*} B\right) \otimes \mu \otimes E$ given by

$\nabla^{\Lambda\left(T^{*} B\right) \otimes \mu \otimes E, e}=\nabla^{\Lambda\left(T^{*} B\right)} \otimes \operatorname{Id}_{\mu \otimes E}+\operatorname{Id}_{\Lambda\left(T^{*} B\right)} \otimes \nabla^{\mu} \otimes \operatorname{Id}_{E}+\operatorname{Id}_{\Lambda\left(T^{*} B\right) \otimes \mu} \otimes \nabla^{E, e}$.

Let the Clifford actions $c, \widehat{c}$ extend to actions on $\Lambda\left(T^{*} B\right) \otimes \mu \otimes E$ by acting as identity on $\mu \otimes E$. Let $\varepsilon$ be the induced $\mathbf{Z}_{2}$-grading operator on $E$, i.e., $\varepsilon=(-1)^{N}$ on $E$. We extend $\varepsilon$ to an action on $\Lambda\left(T^{*} B\right) \otimes \mu \otimes E$ by acting as identity on $\Lambda\left(T^{*} B\right) \otimes \mu$.

Let $\tau \widehat{\otimes} \varepsilon$ define the $\mathbb{Z}_{2}$-grading on $\left(\Lambda\left(T^{*} B\right) \otimes \mu\right) \widehat{\otimes} E$, then

$$
D_{\mathrm{sig}}^{\mu \otimes E}=\sum_{i=1}^{p} c\left(e_{i}\right) \nabla_{e_{i}}^{\Lambda^{*}\left(T^{*} B\right) \otimes \mu \otimes E, e}
$$


defines the twisted signature operator with respect to this $\mathbb{Z}_{2}$-grading. Playing an important role here is its deformation, given by

$$
D_{\mathrm{sig}, u}^{\mu \otimes E}=D_{\mathrm{sig}}^{\mu \otimes E}+\frac{\sqrt{u}}{2}\left(v+v^{*}\right)
$$

with $u>0$, which might be thought of as a quantization of $C_{u}$.

Let $Y_{u}$ be the skew adjoint element in $\operatorname{End}^{\text {odd }}\left(\Lambda^{*}\left(T^{*} B\right) \otimes \mu \otimes E\right)$ defined by (cf. [MZ, (2.18)])

$$
Y_{u}=\frac{1}{2} \sum_{i=1}^{p} c\left(e_{i}\right) \omega\left(E, h^{E}\right)\left(e_{i}\right)+\frac{\sqrt{u}}{2}\left(v^{*}-v\right),
$$

which might be thought of as a quantization of $D_{u}$.

Now following [MZ, Definition 2.3], for any $r \in \mathbf{R}$, define

$$
D_{\mathrm{sig}, u}^{\mu \otimes E}(r)=D_{\mathrm{sig}, u}^{\mu \otimes E}+\sqrt{-1} r Y_{u} .
$$

From (2.15)-(2.18), one has (cf. [MZ, (2.22)])

$$
\begin{array}{r}
D_{\mathrm{sig}, u}^{\mu \otimes E}(r)=\sum_{i=1}^{p} c\left(e_{i}\right)\left(\nabla_{e_{i}}^{\Lambda^{*}\left(T^{*} B\right) \otimes \mu \otimes E, e}+\frac{\sqrt{-1} r}{2} \omega\left(E, h^{E}\right)\left(e_{i}\right)\right) \\
+\frac{\sqrt{u}}{2}\left((1-\sqrt{-1} r) v+(1+\sqrt{-1} r) v^{*}\right) .
\end{array}
$$

Proposition 2.1. We have the following asymptotic expansion

$$
\operatorname{Tr}_{s}\left[D_{\mathrm{sig}, u}^{\mu \otimes E}(r) Y_{u} e^{-t\left(D_{\mathrm{sig}, u}^{\mu \otimes E}(r)\right)^{2}}\right]=c_{0}(u, r)+c_{1}(u, r) t+\cdots
$$

as $t \rightarrow 0$. The expansion is uniform for $(u, r)$ in a compact set.

Proof. We introduce two auxiliary Grassmann variables $z_{1}, z_{2}$ and write

$$
\begin{aligned}
\operatorname{Tr}_{s}\left[D_{\mathrm{sig}, u}^{\mu \otimes E}(r) Y_{u} e^{-t\left(D_{\mathrm{sig}, u}^{\mu \otimes E}(r)\right)^{2}}\right] & = \\
& -t^{-2} \operatorname{Tr}_{s, z_{1}, z_{2}}\left[e^{-t\left(\left[D_{\mathrm{sig}, u}^{\mu \otimes E}(r)\right]^{2}-z_{1} D_{\mathrm{sig}, u}^{\mu \otimes E}(r)-z_{2} Y_{u}\right)}\right] .
\end{aligned}
$$

Here the minus sign comes from the order of the appearance of $z_{1}, z_{2}$ and $D_{\text {sig, } u}^{\mu \otimes E}(r), Y_{u}$.

Applying the standard elliptic theory to the right hand side of (2.21), we derive an asymptotic expansion

$$
\begin{array}{r}
\operatorname{Tr}_{s}\left[D_{\mathrm{sig}, u}^{\mu \otimes E}(r) Y_{u} e^{-t\left(D_{\mathrm{sig}, u}^{\mu \otimes E}(r)\right)^{2}}\right]=c_{-p / 2-2}(u, r) t^{-p / 2-2}+c_{-p / 2-1}(u, r) t^{-p / 2-1}+ \\
\cdots+c_{0}(u, r)+c_{1}(u, r) t+\cdots .
\end{array}
$$

On the other hand, by the Lichnerowicz formula (Cf. (2.30) ) and the same argument as in $[\mathrm{BF}]$, we have

$$
\lim _{t \rightarrow 0} t \operatorname{Tr}_{s}\left[D_{\mathrm{sig}, u}^{\mu \otimes E}(r) Y_{u} e^{-t\left(D_{\mathrm{sig}, u}^{\mu \otimes E}(r)\right)^{2}}\right]=0 .
$$

It follows then that $c_{i}(u, r)=0$ for $-p / 2-2 \leq i \leq-1$. Thus, the asymptotic expansion starts with the constant term. 
Since

$$
\operatorname{Tr}_{s}\left[D_{\mathrm{sig}, u}^{\mu \otimes E}(r) Y_{u} e^{-t\left(D_{\mathrm{sig}, u}^{\mu \otimes E}(r)\right)^{2}}\right]=-\operatorname{Tr}_{s}\left[Y_{u} D_{\mathrm{sig}, u}^{\mu \otimes E}(r) e^{-t\left(D_{\mathrm{sig}, u}^{\mu \otimes E}(r)\right)^{2}}\right]
$$

is also exponentially decaying as $t \rightarrow \infty$, the quantity on the right hand side of the following definition is well-defined.

Definition 2.2. We define

$$
\delta_{u}(E, v)(r)=\int_{0}^{\infty} \operatorname{Tr}_{s}\left[D_{\mathrm{sig}, u}^{\mu \otimes E}(r) Y_{u} e^{-t\left(D_{\mathrm{sig}, u}^{\mu \otimes E}(r)\right)^{2}}\right] d t .
$$

Remark 2.3. If $H^{*}(E, v)=\{0\}$, i.e., $(E, v)$ is acyclic, by [MZ, (2.27)], which we recall as follows,

$$
\left((1-\sqrt{-1} r) v+(1+\sqrt{-1} r) v^{*}\right)^{2}=\left(1+r^{2}\right)\left(v+v^{*}\right)^{2},
$$

(2.19) and proceed as in [BC1], one sees that when $u>0$ is large enough, $D_{\text {sig, } u}^{\mu \otimes E}(r)$ is invertible for fixed $r \in \mathbf{R}$. Since by (2.18) one has

$$
\frac{\partial D_{\mathrm{sig}, u}^{\mu \otimes E}(r)}{\partial r}=\sqrt{-1} Y_{u},
$$

$\frac{\sqrt{-1}}{2} \delta_{u}(E, v)(r)$ is (the imaginary part of) the Bismut-Freed connection form $([\mathrm{BF}$, see also [DF, (3.8)]) over $r$ of the Quillen determinant line bundle of the $r$-family operators $\left\{D_{\text {sig }, u}^{\mu \otimes E}(r)\right\}_{r \in \mathbb{R}}$.

\subsection{Adiabatic limit as $u \rightarrow+\infty$}

We first rewrite $\delta_{u}(E, v)(r)$ as

$$
\delta_{u}(E, v)(r)=\int_{0}^{\infty} \operatorname{Tr}_{s}\left[D_{\epsilon}(r) Y^{\epsilon} e^{-t D_{\epsilon}^{2}(r)}\right] d t,
$$

where $\epsilon=u^{-\frac{1}{2}}$ and

$$
\begin{gathered}
Y^{\epsilon}=\frac{\epsilon}{2} c(\omega)+\frac{1}{2}\left(v^{*}-v\right), \\
D_{\epsilon}(r)=\epsilon D_{\mathrm{sig}, u}^{\mu \otimes E}+\sqrt{-1} r Y^{\epsilon} .
\end{gathered}
$$

We fix a square root of $\sqrt{-1}$ and let $\varphi: \Lambda\left(T^{*} B\right) \rightarrow \Lambda\left(T^{*} B\right)$ be the homomorphism defined by $\varphi: \omega \in \Lambda^{i}\left(T^{*} B\right) \rightarrow(2 \pi \sqrt{-1})^{-i / 2} \omega$. The formulas in what follows will not depend on the choice of the square root of $\sqrt{-1}$.

Let $\mathrm{L}\left(T B, \nabla^{T B}\right)$ be the Hirzebruch characteristic form defined by

$$
\mathrm{L}\left(T B, \nabla^{T B}\right)=\varphi \operatorname{det}^{1 / 2}\left(\frac{R^{T B}}{\tanh \left(R^{T B} / 2\right)}\right),
$$

while $\operatorname{ch}\left(\mu, \nabla^{\mu}\right)$ be the Chern character form defined by

$$
\operatorname{ch}\left(\mu, \nabla^{\mu}\right)=\varphi \operatorname{Tr}\left[\exp \left(-R^{\mu}\right)\right] .
$$


Proposition 2.4. We have

$$
\begin{aligned}
\lim _{\epsilon \rightarrow 0} \operatorname{Tr}_{s}\left[D_{\epsilon}(r) Y^{\epsilon} e^{-t D_{\epsilon}^{2}(r)}\right]=-\int_{B} L\left(T B, \nabla^{T B}\right) \operatorname{ch}\left(\mu, \nabla^{\mu}\right) \\
\cdot \varphi \operatorname{Tr}_{s}\left[t^{-\frac{1}{2}} D_{t}\left(\frac{1}{2}\left(v+v^{*}\right)+\frac{\sqrt{-1} r}{2}\left(v^{*}-v\right)\right) e^{-\left(C_{t}+\sqrt{-1} r D_{t}\right)^{2}}\right] .
\end{aligned}
$$

Proof. As in $[\mathrm{BF}]$ and $[\mathrm{BC1}$, we introduce an auxiliary Grassmann variable $z$ and rewrite

$$
\operatorname{Tr}_{s}\left[D_{\epsilon}(r) Y^{\epsilon} e^{-t D_{\epsilon}^{2}(r)}\right]=-\operatorname{Tr}_{s, z}\left[Y^{\epsilon} t^{-\frac{1}{2}} e^{-t D_{\epsilon}^{2}(r)+z \sqrt{t} D_{\epsilon}(r)}\right],
$$

where for elements of the form $A+z B$ with $A, B$ containing no $z$, we have as in [BF] and [BC1] that $\operatorname{Tr}_{s, z}[A+z B]=\operatorname{Tr}_{s}[B]$.

By (2.16) and (2.25), one has

$$
\begin{aligned}
D_{\epsilon}(r)= & \epsilon D_{\mathrm{sig}}^{\mu \otimes E}+\frac{1}{2}\left(v+v^{*}\right)+\sqrt{-1} r\left(\frac{\epsilon}{2} c(\omega)+\frac{1}{2}\left(v^{*}-v\right)\right) \\
& =\epsilon\left(D_{\mathrm{sig}}^{\mu \otimes E}+\sqrt{-1} r \frac{1}{2} c(\omega)\right)+\frac{1}{2}\left(v+v^{*}\right)+\sqrt{-1} r \frac{1}{2}\left(v^{*}-v\right) .
\end{aligned}
$$

Denote by

$$
V=\frac{1}{2}\left(v+v^{*}\right)+\sqrt{-1} r \frac{1}{2}\left(v^{*}-v\right) .
$$

By Lichnerowicz formula, we have (for simplicity we denote $\nabla=\nabla^{\Lambda^{*}\left(T^{*} B\right) \otimes \mu \otimes E, e}$ )

$$
\begin{gathered}
t D_{\epsilon}^{2}(r)-z \sqrt{t} D_{\epsilon}(r)=t \epsilon^{2}\left(D_{\mathrm{sig}}^{\mu \otimes E}\right)^{2}+t \epsilon^{2}\left[D_{\mathrm{sig}}^{\mu \otimes E}, \sqrt{-1} r \frac{1}{2} c(\omega)\right]+t \epsilon\left[D_{\mathrm{sig}}^{\mu \otimes E}, V\right] \\
+t\left(\sqrt{-1} r \frac{\epsilon}{2} c(\omega)+V\right)^{2}-z \sqrt{t}\left(\epsilon D_{\mathrm{sig}}^{\mu \otimes E}+\sqrt{-1} r \frac{\epsilon}{2} c(\omega)+V\right) \\
=-t\left(\epsilon \nabla_{e_{i}}+\frac{1}{2 \sqrt{t}} z c\left(e_{i}\right)\right)^{2}+\frac{t \epsilon^{2}}{4} k^{T B}+\frac{t \epsilon^{2}}{2} c\left(e_{i}\right) c\left(e_{j}\right) \otimes R^{\mu \otimes E, e}\left(e_{i}, e_{j}\right) \\
+\frac{t \epsilon^{2}}{8} R_{i j k l}^{T B} c\left(e_{i}\right) c\left(e_{j}\right) \hat{c}\left(e_{k}\right) \hat{c}\left(e_{l}\right)+t \epsilon c\left(e_{i}\right) \nabla_{e_{i}} V+\frac{\sqrt{-1} r}{2} t \epsilon^{2} c\left(e_{i}\right) c\left(e_{j}\right) \nabla_{e_{i}} \omega_{j} \\
-t \epsilon^{2} \sqrt{-1} r \sum_{i=1}^{p} \omega\left(e_{i}\right) \nabla_{e_{i}}+t\left(\frac{\sqrt{-1} r}{2} \epsilon c(\omega)+V\right)^{2}-z \sqrt{t} V-z \frac{\sqrt{-1} r}{2} \sqrt{t} \epsilon c(\omega)
\end{gathered}
$$

where $R^{T B}$ is the Riemannian curvature and $k^{T B}$ is the scalar curvature of $g^{T B}$, while $R^{\mu \otimes E, e}$ is the curvature of the connection on $\mu \otimes E$ obtained through $\nabla^{\mu}$ and $\nabla^{E, e}$.

Now we find ourself exactly in the situation of [BC1]. Near any point $x$, take a normal coordinate system $\left\{x_{i}\right\}$ and the associated orthonormal basis $\left\{e_{i}\right\}$. We first conjugate $t D_{\epsilon}^{2}(r)-z \sqrt{t} D_{\epsilon}(r)$ by the exponential $e^{\frac{z \sum_{i=1}^{p} x_{i} c\left(e_{i}\right)}{2 \sqrt{t} \epsilon}}$ 
and then apply the Getzler transformation $G_{\sqrt{t} \epsilon}$. One finds that after these procedures, the operator $t D_{\epsilon}^{2}(r)-z \sqrt{t} D_{\epsilon}(r)$ tends to, as $\epsilon \rightarrow 0$,

$$
\begin{gathered}
-\left(\partial_{i}+\frac{1}{4} R_{i j}^{T B} x_{j}\right)^{2}+\frac{1}{4} R_{k l}^{T B} \hat{c}\left(e_{k}\right) \hat{c}\left(e_{l}\right)+R^{\mu \otimes E, e}+t^{\frac{1}{2}} \nabla V+\frac{\sqrt{-1} r}{2} \nabla \omega \\
+\left(\frac{\sqrt{-1} r}{2} \omega+t^{\frac{1}{2}} V\right)^{2}-z \sqrt{t} V \\
=-\left(\partial_{i}+\frac{1}{4} R_{i j}^{T B} x_{j}\right)^{2}+\frac{1}{4} R_{k l}^{T B} \hat{c}\left(e_{k}\right) \hat{c}\left(e_{l}\right)+R^{\mu} \\
+\left(\nabla^{E, e}+\frac{\sqrt{-1} r}{2} \omega+t^{\frac{1}{2}} V\right)^{2}-z \sqrt{t} V \\
=-\left(\partial_{i}+\frac{1}{4} R_{i j}^{T B} x_{j}\right)^{2}+\frac{1}{4} R_{k l}^{T B} \hat{c}\left(e_{k}\right) \hat{c}\left(e_{l}\right)+\left(C_{t}+\sqrt{-1} r D_{t}\right)^{2} \\
-z \sqrt{t}\left(\frac{1}{2}\left(v+v^{*}\right)+\frac{\sqrt{-1} r}{2}\left(v^{*}-v\right)\right) .
\end{gathered}
$$

On the other hand, by (2.25) it is clear that under the same procedures, $Y^{\epsilon}$ tends to, as $\epsilon \rightarrow 0$,

$$
t^{-\frac{1}{2}}\left(\frac{1}{2} \omega+\frac{\sqrt{t}}{2}\left(v^{*}-v\right)\right)=t^{-\frac{1}{2}} D_{t} .
$$

From (2.27), (2.31) and (2.32), by proceeding the by now standard local index techniques, and keeping in mind that the supertrace in the left hand sides of (2.26) and (2.27) are respect to the $\mathbb{Z}_{2}$-grading defined by $\tau \widehat{\otimes} \varepsilon$, one derives (2.26).

We now examine the terms appearing in the right hand side of (2.26).

By (cf. [MZ, (2.34)])

$$
\left(C_{t}+\sqrt{-1} r D_{t}\right)^{2}=\left(1+r^{2}\right) C_{t}^{2}=-\left(1+r^{2}\right) D_{t}^{2}
$$

and

$$
v+v^{*}=-2 t^{-\frac{1}{2}}\left[N, D_{t}\right], \quad v^{*}-v=-2 t^{-\frac{1}{2}}\left[N, C_{t}\right],
$$

we have

$$
\begin{gathered}
(2.35) \quad-\operatorname{Tr}_{s}\left[t^{-\frac{1}{2}} D_{t}\left(\frac{1}{2}\left(v+v^{*}\right)+\frac{\sqrt{-1} r}{2}\left(v^{*}-v\right)\right) e^{\left.-\left(C_{t}+\sqrt{-1} r D_{t}\right)^{2}\right]}\right. \\
=-\frac{1}{2 \sqrt{t}} \operatorname{Tr}_{s}\left[D_{t}\left(v+v^{*}\right) e^{\left(1+r^{2}\right) D_{t}^{2}}\right]-\frac{\sqrt{-1} r}{2 \sqrt{t}} \operatorname{Tr}_{s}\left[D_{t}\left(v^{*}-v\right) e^{\left(1+r^{2}\right) D_{t}^{2}}\right] \\
=\frac{1}{t} \operatorname{Tr}_{s}\left[D_{t}\left[N, D_{t}\right] e^{\left(1+r^{2}\right) D_{t}^{2}}\right]+\frac{\sqrt{-1} r}{t} \operatorname{Tr}_{s}\left[D_{t}\left[N, C_{t}\right] e^{\left(1+r^{2}\right) D_{t}^{2}}\right] \\
=-\frac{1}{t} \operatorname{Tr}_{s}\left[N D_{t}^{2} e^{\left(1+r^{2}\right) D_{t}^{2}}-D_{t} N D_{t} e^{\left(1+r^{2}\right) D_{t}^{2}}\right]+\frac{\sqrt{-1} r}{t} d \operatorname{Tr}_{s}\left[N D_{t} e^{\left(1+r^{2}\right) D_{t}^{2}}\right],
\end{gathered}
$$

where in the last equality we have used (2.7) (compare also with [MZ, (2.75)]).

We are now ready to prove the following main result of this section. 
Theorem 2.5. Under the assumption that the flat cochain complex $(E, v)$ is acyclic: $H^{*}(E, v)=0$, the following identity holds,

$$
\frac{1}{2} \lim _{u \rightarrow+\infty} \delta_{u}(E, v)(r)=\int_{B} L\left(T B, \nabla^{T B}\right) \operatorname{ch}\left(\mu, \nabla^{\mu}\right) \mathcal{T}_{r}
$$

where

$$
\mathcal{T}_{r}=-\int_{0}^{\infty} \varphi \operatorname{Tr}_{s}\left[N D_{t}^{2} e^{\left(1+r^{2}\right) D_{t}^{2}}\right] \frac{d t}{t}
$$

Proof. First of all, the assumption that $H^{*}(E, v)=0$ implies that the eigenvalues of $D_{\epsilon}(r)$ are uniformly bounded away from zero. Hence the integral in (2.24) is uniformly convergent at $t=\infty$.

We now examine the same issue at $t=0$. From Proposition 2.1, one has

$$
\operatorname{Tr}_{s}\left[D_{\epsilon}(r) Y^{\epsilon} e^{-t\left(D_{\epsilon}(r)\right)^{2}}\right] \quad=\quad c_{0}(\epsilon, r)+c_{1}(\epsilon, r) t+\cdots
$$

We claim that this asymptotic expansion is in fact uniform in $\epsilon$ as $\epsilon \rightarrow 0$ and the coefficients converge to that of asymptotic expansion of the right hand side of (2.26). This can be seen by an argument similar to that of [BC1], which is carried out in detail later for the infinite dimensional case; see the proof of Proposition 3.6.

Our theorem now follows from Proposition 2.4, the equation (2.35) and the above discussion.

Remark 2.6. By Remark 2.3, one sees that under the assumption of Theorem

2.5. for each $r \in \mathbb{R}$, when $u>0$ is large enough, $\frac{\sqrt{-1}}{2} \delta_{u}(E, v)(r)$ is the BismutFreed connection form of the $r$-family of operators $D_{\mathrm{sig}, u}^{\mu \otimes E}(r)$ at $r$. While on the other hand, by comparing the right hand side of (2.37) with [BL] and [MZ], one sees that $\mathcal{T}_{r}$ here gives, up to rescaling, the nonzero degree terms of the BismutLott torsion form ([BL] $)$. Thus, we can say that one obtains the Bismut-Lott torsion form through the adiabatic limit of the Bismut-Freed connection. This is the main philosophy we would like to indicate in this paper.

\section{Sub-signature operators, adiabatic limit and the Bismut-Lott torsion form}

In this section, we deal with the fibration case. We will show that, for an acyclic flat complex vector bundle over a fibered manifold, if we consider the Bismut-Freed connection form $[\mathrm{BF}]$ on the Quillen determinant line bundle associated to the 1-parameter family constructed in [MZ, Section 3], then the Bismut-Lott analytic torsion form [BL] will show up naturally through the adiabatic limit of this connection form. This tautologically answers a question asked implicitly in the original article of Bismut-Lott. 


\subsection{The Bismut-Lott Superconnection}

We first set up the fibration case as an infinite dimensional analog of the case considered in the previous section. Let $\pi: M \rightarrow B$ be a smooth fiber bundle with compact fiber $Z$ of dimension $n$. We denote by $m=\operatorname{dim} M, p=\operatorname{dim} B$. Let $T Z$ be the vertical tangent bundle of the fiber bundle, and let $T^{*} Z$ be its dual bundle.

Let $T M=T^{H} M \oplus T Z$ be a splitting of $T M$. Let $P^{T Z}, P^{T^{H} M}$ denote the projection from $T M$ to $T Z, T^{H} M$. If $U \in T B$, let $U^{H}$ be the lift of $U$ in $T^{H} M$, so that $\pi_{*} U^{H}=U$.

Let $F$ be a flat complex vector bundle on $M$ and let $\nabla^{F}$ denote its flat connection.

Let $E=\oplus_{i=0}^{n} E^{i}$ be the smooth infinite-dimensional $\mathbb{Z}$-graded vector bundle over $B$ whose fiber over $b \in B$ is $C^{\infty}\left(Z_{b},\left(\Lambda\left(T^{*} Z\right) \otimes F\right)_{\mid Z_{b}}\right)$. That is

$$
C^{\infty}\left(B, E^{i}\right)=C^{\infty}\left(M, \Lambda^{i}\left(T^{*} Z\right) \otimes F\right)
$$

Definition 3.1. For $s \in C^{\infty}(B, E)$ and $U$ a vector field on $B$, let $\nabla^{E}$ be a $\mathbb{Z}$-grading preserving connection on $E$ defined by

$$
\nabla_{U}^{E} s=L_{U^{H}} s
$$

where the Lie differential $L_{U^{H}}$ acts on $C^{\infty}(B, E)=C^{\infty}\left(M, \Lambda^{i}\left(T^{*} Z\right) \otimes F\right)$.

If $U_{1}, U_{2}$ are vector fields on $B$, put

$$
T\left(U_{1}, U_{2}\right)=-P^{T Z}\left[U_{1}^{H}, U_{2}^{H}\right] \in C^{\infty}(M, T Z) .
$$

We denote by $i_{T} \in \Omega^{2}\left(B, \operatorname{Hom}\left(E^{\bullet}, E^{\bullet-1}\right)\right)$ the 2-form on $B$ which, to vector fields $U_{1}, U_{2}$ on $B$, assigns the operation of interior multiplication by $T\left(U_{1}, U_{2}\right)$ on $E$.

Let $d^{Z}$ be the exterior differentiation along fibers. We consider $d^{Z}$ to be an element of $C^{\infty}\left(B, \operatorname{Hom}\left(E^{\bullet}, E^{\bullet+1}\right)\right)$. The exterior differentiation operator $d^{M}$, acting on $\Omega(M, F)=C^{\infty}\left(M, \Lambda\left(T^{*} M\right) \otimes F\right)$, has degree 1 and satisfies $\left(d^{M}\right)^{2}=0$. By [BL, Proposition 3.4], we have

$$
d^{M}=d^{Z}+\nabla^{E}+i_{T}
$$

So $d^{M}$ is a flat superconnection of total degree 1 on $E$. We have

$$
\left(d^{Z}\right)^{2}=0, \quad\left[\nabla^{E}, d^{Z}\right]=0 .
$$

Let $g^{T Z}$ be a metric on $T Z$. Let $h^{F}$ be a Hermitian metric on $F$. Let $\nabla^{F *}$ be the adjoint of $\nabla^{F}$ with respect to $h^{F}$. Let $\omega\left(F, h^{F}\right)$ and $\nabla^{F, e}$ be the 1 -form on $M$ and the connection on $F$ defined as in (2.3), (2.8).

Let $o(T Z)$ be the orientation bundle of $T Z$, a flat real line bundle on $M$. Let $d v_{Z}$ be the Riemannian volume form on fibers $Z$ associated to the metric $g^{T Z}$ (Here $d v_{Z}$ is viewed as a section of $\Lambda^{\operatorname{dim} Z}\left(T^{*} Z\right) \otimes o(T Z)$ ). Let $\langle,\rangle_{\Lambda\left(T^{*} Z\right) \otimes F}$ be 
the metric on $\Lambda\left(T^{*} Z\right) \otimes F$ induced by $g^{T Z}, h^{F}$. Then $E$ acquires a Hermitian metric $h^{E}$ such that for $\alpha, \alpha^{\prime} \in C^{\infty}(B, E)$ and $b \in B$,

$$
\left\langle\alpha, \alpha^{\prime}\right\rangle_{h^{E}}(b)=\int_{Z_{b}}\left\langle\alpha, \alpha^{\prime}\right\rangle_{\Lambda\left(T^{*} Z\right) \otimes F} d v_{Z_{b}} .
$$

Let $\nabla^{E *}, d^{Z *},\left(d^{M}\right)^{*},\left(i_{T}\right)^{*}$ be the formal adjoints of $\nabla^{E}, d^{Z}, d^{M}, i_{T}$ with respect to the scalar product $\langle,\rangle_{h^{E}}$. Set

$$
\begin{aligned}
& D^{Z}=d^{Z}+d^{Z *}, \quad \nabla^{E, e}=\frac{1}{2}\left(\nabla^{E}+\nabla^{E *}\right), \\
& \omega\left(E, h^{E}\right)=\nabla^{E *}-\nabla^{E} .
\end{aligned}
$$

Let $N_{Z}$ be the number operator of $E$, i.e. $N_{Z}$ acts by multiplication by $k$ on $C^{\infty}\left(M, \Lambda^{k}\left(T^{*} Z\right) \otimes F\right)$. For $u>0$, set

$$
\begin{aligned}
& C_{u}^{\prime}=u^{N_{Z} / 2} d^{M} u^{-N_{Z} / 2}, \quad C_{u}^{\prime \prime}=u^{-N_{Z} / 2}\left(d^{M}\right)^{*} u^{N_{Z} / 2}, \\
& C_{u}=\frac{1}{2}\left(C_{u}^{\prime}+C_{u}^{\prime \prime}\right), \quad D_{u}=\frac{1}{2}\left(C_{u}^{\prime \prime}-C_{u}^{\prime}\right) .
\end{aligned}
$$

Then $C_{u}^{\prime \prime}$ is the adjoint of $C_{u}^{\prime}$ with respect to $h^{E}$. Moreover, $C_{u}$ is a superconnection on $E$ and $D_{u}$ is an odd element of $C^{\infty}(B, \operatorname{End}(E))$, and

$$
C_{u}^{2}=-D_{u}^{2}, \quad\left[C_{u}, D_{u}\right]=0 .
$$

Let $g^{T B}$ be a Riemannian metric on $T B$. Then $g^{T M}=g^{T Z} \oplus \pi^{*} g^{T B}$ is a metric on $T M$. Let $\nabla^{T M}, \nabla^{T B}$ denote the corresponding Levi-Civita connections on $T M, T B$. Put $\nabla^{T Z}=P^{T Z} \nabla^{T M}$, a connection on $T Z$. As shown in [B, Theorem 1.9], $\nabla^{T Z}$ is independent of the choice of $g^{T B}$. Then ${ }^{0} \nabla=\nabla^{T Z} \oplus \pi^{*} \nabla^{T B}$ is also a connection on $T M$. Let $S=\nabla^{T M}{ }^{0} \nabla$. By [B, Theorem 1.9], $\langle S(\cdot) \cdot, \cdot\rangle_{g^{T M}}$ is a tensor independent of $g^{T B}$. Moreover, for $U_{1}, U_{2} \in T B, X, Y \in T Z$,

$$
\begin{aligned}
& \left\langle S\left(U_{1}^{H}\right) X, U_{2}^{H}\right\rangle_{g^{T M}}=-\left\langle S\left(U_{1}^{H}\right) U_{2}^{H}, X\right\rangle_{g^{T M}} \\
& =\left\langle S(X) U_{1}^{H}, U_{2}^{H}\right\rangle_{g^{T M}}=\frac{1}{2}\left\langle T\left(U_{1}^{H}, U_{2}^{H}\right), X\right\rangle_{g^{T M}}, \\
& \left\langle S(X) Y, U_{1}^{H}\right\rangle_{g^{T M}}=-\left\langle S(X) U_{1}^{H}, Y\right\rangle_{g^{T M}}=\frac{1}{2}\left(L_{U_{1}^{H}} g^{T Z}\right)(X, Y),
\end{aligned}
$$

and all other terms are zero.

Let $\left\{f_{\alpha}\right\}_{\alpha=1}^{p}$ be an orthonormal basis of $T B$, set $\left\{f^{\alpha}\right\}_{\alpha=1}^{p}$ the dual basis of $T^{*} B$. In the following, it's convenient to identify $f_{\alpha}$ with $f_{\alpha}^{H}$. Let $\left\{e_{i}\right\}_{i=1}^{n}$ be an orthonormal basis of $\left(T Z, g^{T Z}\right)$. We define a horizontal 1 -form $k$ on $M$ by

$$
k\left(f_{\alpha}\right)=-\sum_{i}\left\langle S\left(e_{i}\right) e_{i}, f_{\alpha}\right\rangle .
$$

Set

$$
\begin{aligned}
& c(T)=\frac{1}{2} \sum_{\alpha, \beta} f^{\alpha} \wedge f^{\beta} c\left(T\left(f_{\alpha}, f_{\beta}\right)\right), \\
& \widehat{c}(T)=\frac{1}{2} \sum_{\alpha, \beta} f^{\alpha} \wedge f^{\beta} \widehat{c}\left(T\left(f_{\alpha}, f_{\beta}\right)\right) .
\end{aligned}
$$


Let $\nabla^{\Lambda\left(T^{*} Z\right)}$ be the connection on $\Lambda\left(T^{*} Z\right)$ induced by $\nabla^{T Z}$. Let $\nabla^{T Z \otimes F, e}$ be the connection on $\Lambda\left(T^{*} Z\right) \otimes F$ induced by $\nabla^{\Lambda\left(T^{*} Z\right)}, \nabla^{F, e}$. Then by [BL, (3.36), (3.37), (3.42)],

$$
\begin{aligned}
& D^{Z}=\sum_{j} c\left(e_{j}\right) \nabla_{e_{j}}^{T Z \otimes F, e}-\frac{1}{2} \sum_{j} \widehat{c}\left(e_{j}\right) \omega\left(F, h^{F}\right)\left(e_{j}\right), \\
& d^{Z *}-d^{Z}=-\sum_{j} \widehat{c}\left(e_{j}\right) \nabla_{e_{j}}^{T Z \otimes F, e}+\frac{1}{2} \sum_{j} c\left(e_{j}\right) \omega\left(F, h^{F}\right)\left(e_{j}\right), \\
& \nabla^{E, e}=\sum_{\alpha} f^{\alpha}\left(\nabla_{f_{\alpha}}^{T Z \otimes F, e}+\frac{1}{2} k\left(f_{\alpha}\right)\right), \\
& \omega\left(E, h^{E}\right)=\sum_{\alpha} f^{\alpha}\left(\sum_{i, j}\left\langle S\left(e_{i}\right) e_{j}, f_{\alpha}\right\rangle c\left(e_{i}\right) \widehat{c}\left(e_{j}\right)+\omega\left(F, h^{F}\right)\left(f_{\alpha}\right)\right) .
\end{aligned}
$$

By [BL, Proposition 3.9], one has

$$
\begin{aligned}
C_{u} & =\frac{\sqrt{u}}{2} D^{Z}+\nabla^{E, e}-\frac{1}{2 \sqrt{u}} c(T), \\
D_{u} & =\frac{\sqrt{u}}{2}\left(d^{Z *}-d^{Z}\right)+\frac{1}{2} \omega\left(E, h^{E}\right)-\frac{1}{2 \sqrt{u}} \widehat{c}(T) .
\end{aligned}
$$

\subsection{Deformed sub-signature operators on a fibered manifold}

We assume now that $T B$ is oriented.

Let $\left(\mu, h^{\mu}\right)$ be a Hermitian complex vector bundle over $B$ carrying a Hermitian connection $\nabla^{\mu}$.

Let $N_{B}, N_{M}$ be the number operators on $\Lambda\left(T^{*} B\right), \Lambda\left(T^{*} M\right)$, i.e. they act as multiplication by $k$ on $\Lambda^{k}\left(T^{*} B\right), \Lambda^{k}\left(T^{*} M\right)$ respectively. Then $N_{M}=N_{B}+N_{Z}$.

Let $\nabla^{\Lambda\left(T^{*} M\right)}$ be the connection on $\Lambda\left(T^{*} M\right)$ canonically induced from $\nabla^{T M}$. Let $\nabla^{\Lambda\left(T^{*} M\right) \otimes \pi^{*} \mu \otimes F}$ (resp. $\nabla^{\Lambda\left(T^{*} M\right) \otimes \pi^{*} \mu \otimes F, e}$ ) be the tensor product connection on $\Lambda\left(T^{*} M\right) \otimes \pi^{*} \mu \otimes F$ induced by $\nabla^{\Lambda\left(T^{*} M\right)}, \pi^{*} \nabla^{\mu}$ and $\nabla^{F}$ (resp. $\nabla^{F, e}$ ).

Let $\left\{e_{a}\right\}_{a=1}^{m}$ be an orthonormal basis of $T M$, and its dual basis $\left\{e^{a}\right\}_{a=1}^{m}$. Let $\left\{f_{\alpha}\right\}_{\alpha=1}^{p}$ be an oriented orthonormal basis of $T B$. Set

$$
\begin{aligned}
& \tau(T B)=(\sqrt{-1})^{\frac{p(p+1)}{2}} c\left(f_{1}^{H}\right) \cdots c\left(f_{p}^{H}\right), \\
& \tau=(-1)^{N_{Z}} \tau(T B) .
\end{aligned}
$$

Then the operators $\tau(T B), \tau$ act naturally on $\Lambda\left(T^{*} M\right)$, and

$$
\tau(T B)^{2}=\tau^{2}=1 .
$$

Let $d^{\nabla^{\mu}}: \Omega^{a}\left(M, \pi^{*} \mu \otimes F\right) \rightarrow \Omega^{a+1}\left(M, \pi^{*} \mu \otimes F\right)$ be the unique extension of $\nabla^{\mu}, \nabla^{F}$ which satisfies the Leibniz rule. Let $d^{\nabla^{\mu} *}$ be the adjoint of $d^{\nabla^{\mu}}$ with respect to the scalar product $\langle,\rangle_{\Omega\left(M, \pi^{*} \mu \otimes F\right)}$ on $\Omega\left(M, \pi^{*} \mu \otimes F\right)$ induced by 
$g^{T M}, h^{\mu}, h^{F}$ as in (3.6). As in [BZ, (4.26), (4.27)], we have

$$
\begin{aligned}
& d^{\nabla^{\mu}}=\sum_{a} e^{a} \wedge \nabla_{e_{a}}^{\Lambda\left(T^{*} M\right) \otimes \pi^{*} \mu \otimes F} \\
& d^{\nabla^{\mu_{*}}}=-\sum_{a} i_{e_{a}} \wedge\left(\nabla_{e_{a}}^{\Lambda\left(T^{*} M\right) \otimes \pi^{*} \mu \otimes F}+\omega\left(F, h^{F}\right)\left(e_{a}\right)\right) .
\end{aligned}
$$

Following [Z1], let $\widetilde{\nabla}^{\Lambda\left(T^{*} M\right)}$ be the Hermitian connection on $\Lambda\left(T^{*} M\right)$ defined by (cf. [Z1, (1.21)])

$$
\widetilde{\nabla}_{X}^{\Lambda\left(T^{*} M\right)}=\nabla_{X}^{\Lambda\left(T^{*} M\right)}-\frac{1}{2} \sum_{\alpha=1}^{p} \widehat{c}\left(P^{T Z} S(X) f_{\alpha}\right) \widehat{c}\left(f_{\alpha}\right), \quad X \in T M .
$$

Let $\widetilde{\nabla}^{e}$ be the tensor product connection on $\Lambda\left(T^{*} M\right) \otimes \pi^{*} \mu \otimes F$ induced by $\widetilde{\nabla}^{\Lambda\left(T^{*} M\right)}, \pi^{*} \nabla^{\mu}$ and $\nabla^{F, e}$. Following [MZ, (3.23)], for any $r \in \mathbb{R}$, set

$$
\begin{gathered}
D^{\pi^{*} \mu \otimes F}=\sum_{a=1}^{m} c\left(e_{a}\right) \widetilde{\nabla}_{e_{a}}^{e}-\frac{1}{2} \sum_{i=1}^{n} \widehat{c}\left(e_{i}\right) \omega\left(F, h^{F}\right)\left(e_{i}\right), \\
\widehat{D}^{\pi^{*} \mu \otimes F}=-\sum_{i=1}^{n} \widehat{c}\left(e_{i}\right) \widetilde{\nabla}_{e_{i}}^{e}+\frac{1}{2} \sum_{a=1}^{m} c\left(e_{a}\right) \omega\left(F, h^{F}\right)\left(e_{a}\right) \\
-\frac{1}{4} \sum_{\alpha, \beta=1}^{p} \widehat{c}\left(T\left(f_{\alpha}, f_{\beta}\right)\right) \widehat{c}\left(f_{\alpha}\right) \widehat{c}\left(f_{\beta}\right), \\
D^{\pi^{*} \mu \otimes F}(r)=D^{\pi^{*} \mu \otimes F}+\sqrt{-1} r \widehat{D}^{\pi^{*} \mu \otimes F} .
\end{gathered}
$$

From (3.19), the operators $D^{\pi^{*}} \mu \otimes F, D^{\pi^{*}} \mu \otimes F(r)$ are formally self-adjoint first order elliptic operators, and $\widehat{D}^{\pi^{*} \mu \otimes F}$ is a skew-adjoint first order differential operator. Moreover, the operator $D^{\pi^{*} \mu \otimes F}$ is locally of Dirac type.

By [MZ, (3.20) and Proposition 3.4], one has

$$
\begin{aligned}
& D^{\pi^{*} \mu \otimes F}=\frac{1}{2}\left[\left(d^{\nabla^{\mu}}+d^{\nabla^{\mu} *}\right)+(-1)^{p+1} \tau\left(d^{\nabla^{\mu}}+d^{\nabla^{\mu} *}\right) \tau\right], \\
& \widehat{D}^{\pi^{*} \mu \otimes F}=\frac{1}{2}\left[\left(d^{\nabla^{\mu_{*}}}-d^{\nabla^{\mu}}\right)+(-1)^{p+1} \tau\left(d^{\nabla^{\mu_{*}}}-d^{\nabla^{\mu}}\right) \tau\right],
\end{aligned}
$$

which partly explains the motivation of introducing these operators (compare with (2.25)).

By (3.15), (3.16) and (3.20), one verifies (cf. [MZ, (3.28)])

$$
\tau D^{\pi^{*} \mu \otimes F}=(-1)^{p+1} D^{\pi^{*} \mu \otimes F} \tau, \quad \tau \widehat{D}^{\pi^{*} \mu \otimes F}=(-1)^{p+1} \widehat{D}^{\pi^{*} \mu \otimes F} \tau .
$$

Remark 3.2. It is important to note that by (3.21), when $p=\operatorname{dim} B$ is even, both $D^{\pi^{*} \mu \otimes F}$ and $\widehat{D}^{\pi^{*} \mu \otimes F}$ anti-commute with $\tau$.

Remark 3.3. When $\mu=F=\mathbf{C}$ and $p=\operatorname{dim} B$ is even, $D^{\pi^{*} \mu \otimes F}$ has been constructed in [Z1] and [Z2], where it is called the sub-signature operator. 


\subsection{Bismut-Freed connection of the deformed family}

We assume that $p=\operatorname{dim} B$ is even. Moreover, we make the following technical assumption.

Technical assumption. The flat vector bundle $F$ over $M$ is fiberwise acyclic, that is $H^{*}\left(Z_{b},\left.F\right|_{Z_{b}}\right)=\{0\}$ on each fiber $Z_{b}, b \in B$.

For any $\varepsilon>0$, we change $g^{T B}$ to $\frac{1}{\varepsilon} g^{T B}$ and do everything again for $g_{\varepsilon}^{T M}=$ $g^{T Z} \oplus \frac{1}{\varepsilon} \pi^{*} g^{T B}$. We will use a subscript $\varepsilon$ to denote the resulting objects.

For any $r \in \mathbb{R}$, one verifies directly that the coefficients of $\frac{1}{\sqrt{\varepsilon}}$ in $\frac{1}{\sqrt{\varepsilon}} D_{\varepsilon}^{\pi^{*} \mu \otimes F}(r)$ is given by $d^{Z}+d^{Z *}-\sqrt{-1} r\left(d^{Z}-d^{Z *}\right)$. Since

$$
\left(d^{Z}+d^{Z *}-\sqrt{-1} r\left(d^{Z}-d^{Z *}\right)\right)^{2}=\left(1+r^{2}\right)\left(d^{Z}+d^{Z *}\right)^{2},
$$

by proceeding as in [BC1], one sees that when $\varepsilon>0$ is small enough, $D_{\varepsilon}^{\pi^{*} \mu \otimes F}(r)$ is invertible near $r$. In fact, the eigenvalues of $D_{\varepsilon}^{\pi^{*} \mu \otimes F}(r)$ are uniformly bounded away from zero.

Consider now $D_{\varepsilon}^{\pi^{*} \mu \otimes F}(r)$ as an $r$-family which anti-commutes with the $\mathbb{Z}_{2^{-}}$ grading defined by $\tau$.

Then one can construct the Quillen determinant line bundle over $r$ and the associated Bismut-Freed connection on it (cf. $[\mathrm{BF}]$ ). Moreover, by the above discussion and by $[\mathrm{BF}, 3.8]$, we know that when $\varepsilon>0$ is small enough, the imaginary part of the Bismut-Freed connection form is given by

$$
\begin{aligned}
& \frac{1}{2 \sqrt{-1}} \text { F.P. } \int_{0}^{+\infty} \operatorname{Tr}_{s}\left[D_{\varepsilon}^{\pi^{*} \mu \otimes F}(r) \frac{\partial D_{\varepsilon}^{\pi^{*} \mu \otimes F}(r)}{\partial r} e^{-t\left(D_{\varepsilon}^{\pi^{*} \mu \otimes F}(r)\right)^{2}}\right] d t \\
= & \frac{1}{2} \text { F.P. } \int_{0}^{+\infty} \operatorname{Tr}_{s}\left[D_{\varepsilon}^{\pi^{*} \mu \otimes F}(r) \widehat{D}_{\varepsilon}^{\pi^{*} \mu \otimes F} e^{-t\left(D_{\varepsilon}^{\pi^{*} \mu \otimes F}(r)\right)^{2}}\right] d t \\
= & -\frac{1}{2} \text { F.P. } \int_{0}^{+\infty} \operatorname{Tr}_{s}\left[\widehat{D}_{\varepsilon}^{\pi^{*} \mu \otimes F} D_{\varepsilon}^{\pi^{*} \mu \otimes F}(r) e^{-t\left(D_{\varepsilon}^{\pi^{*} \mu \otimes F}(r)\right)^{2}}\right] d t,
\end{aligned}
$$

where the supertrace is with respect to $\tau$ and 'F.P.' means taking the finite part of the (divergent) integral. As usual we use the zeta function regularization. Thus, we define

$$
\delta_{\varepsilon}(F, r)(s)=-\frac{1}{2 \Gamma(s)} \int_{0}^{+\infty} t^{s} \operatorname{Tr}_{s}\left[\widehat{D}_{\varepsilon}^{\pi^{*} \mu \otimes F} D_{\varepsilon}^{\pi^{*} \mu \otimes F}(r) e^{-t\left(D_{\varepsilon}^{\pi^{*} \mu \otimes F}(r)\right)^{2}}\right] d t .
$$

Remark. Note that we have built the factor $\frac{1}{2}$ into the definition (unlike the finite dimensional case).

In the next subsection we will study the asymptotic expansions of the integrand in (3.23) which implies that the integral, convergent for $\Re s$ sufficiently 
large, has meromorphic continuation to the whole complex plane with $s=0$ a regular point. Therefore we define our invariant by

$$
\delta_{\varepsilon}(F)(r)=\delta_{\varepsilon}(F, r)^{\prime}(0) .
$$

Also in the next section we will compute the adiabatic limit of $\delta_{\varepsilon}(F)(r)$ as $\varepsilon \rightarrow 0$.

We remark that the definition of $\delta_{\varepsilon}(F)(r)$ does not make use of the technical assumption $H^{*}\left(Z_{b},\left.F\right|_{Z_{b}}\right)=\{0\}$.

\subsection{The adiabatic limit and the torsion form}

We begin with a lemma.

Lemma 3.4. $\widehat{D}^{\pi^{*} \mu \otimes F}$ is the quantization of $D_{4}$; namely, it is obtained by replacing the horizontal differential forms in $D_{4}$ by the corresponding Clifford multiplications. Similarly, $D^{\pi^{*} \mu \otimes F}$ is the quantization of $C_{4}$.

Proof. By (3.19),

$\widehat{D}^{\pi^{*} \mu \otimes F}=-\sum_{i=1}^{n} \widehat{c}\left(e_{i}\right) \widetilde{\nabla}_{e_{i}}^{e}+\frac{1}{2} \sum_{a=1}^{m} c\left(e_{a}\right) \omega\left(F, h^{F}\right)\left(e_{a}\right)-\frac{1}{4} \sum_{\alpha, \beta=1}^{p} \widehat{c}\left(T\left(f_{\alpha}, f_{\beta}\right)\right) \widehat{c}\left(f_{\alpha}\right) \widehat{c}\left(f_{\beta}\right)$,

where the connection $\widetilde{\nabla}^{\Lambda\left(T^{*} M\right)}$ is defined by (3.18). Thus, we now look at the connection in a bit more detail. Since $\nabla^{T M}={ }^{0} \nabla+S$ and ${ }^{0} \nabla=\nabla^{T Z} \oplus \pi^{*} \nabla^{T B}$, we find (for simplicity, we denote $S_{i j \alpha}=\left\langle S\left(e_{i}\right) e_{j}, f_{\alpha}\right\rangle$ and so on)

$$
\begin{aligned}
\nabla_{e_{i}}^{\Lambda\left(T^{*} M\right)}= & \nabla_{e_{i}}^{\Lambda\left(T^{*} Z\right)}-\frac{1}{4}\left[S_{i j \alpha}\left(\widehat{c}\left(e_{j}\right) \widehat{c}\left(f_{\alpha}\right)-c\left(e_{j}\right) c\left(f_{\alpha}\right)+c\left(e_{j}\right) \widehat{c}\left(f_{\alpha}\right)-\widehat{c}\left(e_{j}\right) c\left(f_{\alpha}\right)\right)\right. \\
& +S_{i \alpha j}\left(\widehat{c}\left(f_{\alpha}\right) \widehat{c}\left(e_{j}\right)-c\left(f_{\alpha}\right) c\left(e_{j}\right)+c\left(f_{\alpha}\right) \widehat{c}\left(e_{j}\right)-\widehat{c}\left(f_{\alpha}\right) c\left(e_{j}\right)\right) \\
& \left.+S_{i \alpha \beta}\left(\widehat{c}\left(f_{\alpha}\right) \widehat{c}\left(f_{\beta}\right)-c\left(f_{\alpha}\right) c\left(f_{\beta}\right)+c\left(f_{\alpha}\right) \widehat{c}\left(f_{\beta}\right)-\widehat{c}\left(f_{\alpha}\right) c\left(f_{\beta}\right)\right)\right] \\
= & \nabla_{e_{i}}^{\Lambda\left(T^{*} Z\right)}-\frac{1}{2} S_{i \alpha j}\left[\widehat{c}\left(f_{\alpha}\right) \widehat{c}\left(e_{j}\right)-c\left(f_{\alpha}\right) c\left(e_{j}\right)\right] \\
& -\frac{1}{4} S_{i \alpha \beta}\left[\widehat{c}\left(f_{\alpha}\right) \widehat{c}\left(f_{\beta}\right)-c\left(f_{\alpha}\right) c\left(f_{\beta}\right)+c\left(f_{\alpha}\right) \widehat{c}\left(f_{\beta}\right)-\widehat{c}\left(f_{\alpha}\right) c\left(f_{\beta}\right)\right]
\end{aligned}
$$

Hence

$$
\begin{aligned}
\widetilde{\nabla}_{e_{i}}^{\Lambda\left(T^{*} M\right)=} & \nabla_{e_{i}}^{\Lambda\left(T^{*} Z\right)}-\frac{1}{2} S_{i j \alpha} c\left(f_{\alpha}\right) c\left(e_{j}\right) \\
& -\frac{1}{4} S_{i \alpha \beta}\left[\widehat{c}\left(f_{\alpha}\right) \widehat{c}\left(f_{\beta}\right)-c\left(f_{\alpha}\right) c\left(f_{\beta}\right)+c\left(f_{\alpha}\right) \widehat{c}\left(f_{\beta}\right)-\widehat{c}\left(f_{\alpha}\right) c\left(f_{\beta}\right)\right] .
\end{aligned}
$$

Therefore,

$$
\begin{aligned}
\widehat{c}\left(e_{i}\right) \widetilde{\nabla}_{e_{i}}^{\Lambda\left(T^{*} M\right)}= & \widehat{c}\left(e_{i}\right) \nabla_{e_{i}}^{\Lambda\left(T^{*} Z\right)} \\
& -\frac{1}{4} S_{i \alpha \beta} \widehat{c}\left(e_{i}\right)\left[\widehat{c}\left(f_{\alpha}\right) \widehat{c}\left(f_{\beta}\right)-c\left(f_{\alpha}\right) c\left(f_{\beta}\right)+c\left(f_{\alpha}\right) \widehat{c}\left(f_{\beta}\right)-\widehat{c}\left(f_{\alpha}\right) c\left(f_{\beta}\right)\right] .
\end{aligned}
$$


And so

$$
\begin{aligned}
\widehat{c}\left(e_{i}\right) \widetilde{\nabla}_{e_{i}}^{e}= & \widehat{c}\left(e_{i}\right) \nabla_{e_{i}}^{T Z \otimes F, e}-\frac{1}{8} \widehat{c}\left(T\left(f_{\alpha}, f_{\beta}\right)\right) \widehat{c}\left(f_{\alpha}\right) \widehat{c}\left(f_{\beta}\right)+\frac{1}{8} \widehat{c}\left(T\left(f_{\alpha}, f_{\beta}\right)\right) c\left(f_{\alpha}\right) c\left(f_{\beta}\right) \\
& -\frac{1}{8} \widehat{c}\left(T\left(f_{\alpha}, f_{\beta}\right)\right)\left[c\left(f_{\alpha}\right) \widehat{c}\left(f_{\beta}\right)-\widehat{c}\left(f_{\alpha}\right) c\left(f_{\beta}\right)\right] .
\end{aligned}
$$

The last term here, $-\frac{1}{8} \widehat{c}\left(T\left(f_{\alpha}, f_{\beta}\right)\right)\left[c\left(f_{\alpha}\right) \widehat{c}\left(f_{\beta}\right)-\widehat{c}\left(f_{\alpha}\right) c\left(f_{\beta}\right)\right]$, vanishes by the antisymmetry. Using the formula above together with (3.13), (3.14), (3.19), we prove our lemma. (The other case is dealt with similarly.)

Proposition 3.5. We have

$$
\begin{array}{r}
\lim _{\epsilon \rightarrow 0} \operatorname{Tr}_{s}\left[\widehat{D}_{\varepsilon}^{\pi^{*} \mu \otimes F} D_{\varepsilon}^{\pi^{*} \mu \otimes F}(r) e^{-t\left(D_{\varepsilon}^{\pi^{*} \mu \otimes F}(r)\right)^{2}}\right]=-\int_{B} L\left(T B, \nabla^{T B}\right) \operatorname{ch}\left(\mu, \nabla^{\mu}\right) \\
\cdot \varphi \operatorname{Tr}_{s}\left[t^{-\frac{1}{2}} D_{4 t} \frac{\partial}{\partial \sqrt{t}}\left(C_{4 t}+\sqrt{-1} r D_{4 t}\right) e^{-\left(1+r^{2}\right) C_{4 t}^{2}}\right] .
\end{array}
$$

Proof. Again, we introduce an auxiliary Grassmann variable $z$ and rewrite

$$
\begin{aligned}
& \operatorname{Tr}_{s}\left[\widehat{D}_{\varepsilon}^{\pi^{*} \mu \otimes F} D_{\varepsilon}^{\pi^{*} \mu \otimes F}(r) e^{-t\left(D_{\varepsilon}^{\pi^{*} \mu \otimes F}(r)\right)^{2}}\right]= \\
&-\operatorname{Tr}_{s, z}\left[t^{-\frac{1}{2}} \widehat{D}_{\varepsilon}^{\pi^{*} \mu \otimes F} e^{-t\left(D_{\varepsilon}^{\pi^{*} \mu \otimes F}(r)\right)^{2}+z \sqrt{t} D_{\varepsilon}^{\pi^{*} \mu \otimes F}(r)}\right] .
\end{aligned}
$$

The following Lichnerowicz formula was proved in [Z1, Theorem 1.1].

$$
\begin{gathered}
\left(D^{\pi^{*} \mu \otimes F}\right)^{2}=-\widetilde{\Delta}^{e}+\frac{K}{4}+\frac{1}{2} \sum_{a, b=1}^{m} c\left(e_{a}\right) c\left(e_{b}\right)\left(\widehat{R}^{e}+\pi^{*} R^{\mu}\right)\left(e_{a}, e_{b}\right) \\
+\frac{1}{4} \sum_{i=1}^{n}\left(\omega\left(F, h^{F}\right)\left(e_{i}\right)\right)^{2}+\frac{1}{8} \sum_{i, j=1}^{n} \widehat{c}\left(e_{i}\right) \widehat{c}\left(e_{j}\right)\left(\omega\left(F, h^{F}\right)\right)^{2}\left(e_{i}, e_{j}\right) \\
-\frac{1}{2} \sum_{a=1}^{m} c\left(e_{a}\right)\left[\sum_{i=1}^{n} \widehat{c}\left(e_{i}\right) \nabla_{e_{a}}^{T M \otimes F, e} \omega\left(F, h^{F}\right)\left(e_{i}\right)\right. \\
\left.+\sum_{\alpha=1}^{p} \widehat{c}\left(f_{\alpha}\right) \omega\left(F, h^{F}\right)\left(P^{T Z} S\left(e_{a}\right) f_{\alpha}\right)\right] .
\end{gathered}
$$


Similarly, the following formulas are shown in [MZ, Proposition 3.6].

$$
\begin{array}{r}
\left(\widehat{D}^{\pi^{*} \mu \otimes F}\right)^{2}=\sum_{i=1}^{n}\left(\left(\widetilde{\nabla}_{e_{i}}^{e}\right)^{2}-\widetilde{\nabla}_{\nabla_{e_{i}}^{T M} e_{i}}^{e}\right)+\frac{1}{2} \sum_{i, j=1}^{n} \widehat{c}\left(e_{i}\right) \widehat{c}\left(e_{j}\right)\left(\widetilde{\nabla}^{e}\right)^{2}\left(e_{i}, e_{j}\right) \\
+\frac{1}{4} \sum_{i=1}^{n} \widehat{c}\left(e_{i}\right)\left[\widetilde{\nabla}_{e_{i}}^{e}, \sum_{\alpha, \beta=1}^{p} \widehat{c}\left(T\left(f_{\alpha}, f_{\beta}\right)\right) \widehat{c}\left(f_{\alpha}\right) \widehat{c}\left(f_{\beta}\right)\right] \\
+\frac{1}{2} \sum_{\alpha, \beta=1}^{p} \widehat{c}\left(f_{\alpha}\right) \widehat{c}\left(f_{\beta}\right) \widetilde{\nabla}_{T\left(f_{\alpha}, f_{\beta}\right)}^{e}-\frac{1}{2} \sum_{i=1}^{n} \sum_{a=1}^{m} \widehat{c}\left(e_{i}\right) c\left(e_{a}\right)\left(\nabla_{e_{i}}^{T M \otimes F, e} \omega\left(F, h^{F}\right)\right)\left(e_{a}\right) \\
-\frac{1}{4} \sum_{a=1}^{m}\left(\omega\left(F, h^{F}\right)\left(e_{a}\right)\right)^{2}+\frac{1}{8} \sum_{a, b=1}^{m} c\left(e_{a}\right) c\left(e_{b}\right)\left(\omega\left(F, h^{F}\right)\right)^{2}\left(e_{a}, e_{b}\right) \\
+\frac{1}{16}\left(\sum_{\alpha, \beta=1}^{p} \widehat{c}\left(T\left(f_{\alpha}, f_{\beta}\right)\right) \widehat{c}\left(f_{\alpha}\right) \widehat{c}\left(f_{\beta}\right)\right)^{2},
\end{array}
$$

$$
\begin{array}{r}
{\left[D^{\pi^{*} \mu \otimes F}, \widehat{D}^{\pi^{*} \mu \otimes F}\right]=-\sum_{a=1}^{m} \sum_{i=1}^{n} c\left(e_{a}\right) \widehat{c}\left(e_{i}\right)\left(\widehat{R}^{e}+\pi^{*} R^{\mu}+\frac{1}{4} \omega\left(F, h^{F}\right)^{2}\right)\left(e_{a}, e_{i}\right)} \\
-\sum_{\alpha=1}^{p} \omega\left(F, h^{F}\right)\left(f_{\alpha}\right) \widetilde{\nabla}_{f_{\alpha}}^{e}+\frac{1}{4} \sum_{\alpha, \beta=1}^{p} \omega\left(F, h^{F}\right)\left(T\left(f_{\alpha}, f_{\beta}\right)\right) \widehat{c}\left(f_{\alpha}\right) \widehat{c}\left(f_{\beta}\right) \\
+\frac{1}{4} \sum_{a=1}^{m} c\left(e_{a}\right)\left[\widetilde{\nabla}_{e_{a}}^{e}, \sum_{\alpha, \beta=1}^{p} \widehat{c}\left(T\left(f_{\alpha}, f_{\beta}\right)\right) \widehat{c}\left(f_{\alpha}\right) \widehat{c}\left(f_{\beta}\right)\right] .
\end{array}
$$

These formulas show that there are no second order fiberwise differentiation in $\left(\widehat{D}^{\pi^{*} \mu \otimes F}\right)^{2}$ and $\left[D^{\pi^{*} \mu \otimes F}, \widehat{D}^{\pi^{*} \mu \otimes F}\right]$. Therefore one can apply the standard Getzler rescaling to $\left(\widehat{D}_{s, \varepsilon}^{\pi^{*} \mu \otimes F}\right)^{2}$ and $\left[D_{s, \varepsilon}^{\pi^{*} \mu \otimes F}, \widehat{D}_{s, \varepsilon}^{\pi^{*} \mu \otimes F}\right]$ with no problem and all terms converge as $\epsilon \rightarrow 0$.

On the other hand, in [Z1, Proposition 2.2], Zhang formulated a Lichnerowicz type formula for $t\left(D_{s, \varepsilon}^{\pi^{*} \mu \otimes F}\right)^{2}-z \sqrt{t} D_{s, \varepsilon}^{\pi^{*} \mu \otimes F}$. The only singular term (for Getzler's rescaling) as $\varepsilon \rightarrow 0$ appears in

$$
-t \varepsilon \sum_{\alpha}\left(\widetilde{\nabla}_{f_{\alpha}}+\frac{\sqrt{\varepsilon}}{2} \sum_{i, \beta}\left\langle S\left(f_{\alpha}\right) e_{i}, f_{\beta}\right\rangle c\left(e_{i}\right) c\left(f_{\beta}\right)+\frac{z c\left(f_{\alpha}\right)}{2 \sqrt{t \varepsilon}}\right)^{2} .
$$

This singular term can be easily eliminated by the exponential transform, namely conjugating by the exponential

$$
e^{\frac{z \sum_{\alpha=1}^{p} y_{\alpha} c\left(f_{\alpha}\right)}{2 \sqrt{t \epsilon}}} .
$$

We then do the Getzler rescaling $G_{\sqrt{t \varepsilon}}$ :

$$
y_{\alpha} \rightarrow \sqrt{t \varepsilon} y_{\alpha}, \quad \nabla_{f_{\alpha}} \rightarrow \frac{1}{\sqrt{t \varepsilon}} \nabla_{f_{\alpha}}, \quad c\left(f_{\alpha}\right) \rightarrow \frac{1}{\sqrt{t \varepsilon}} f^{\alpha} \wedge-\sqrt{t \varepsilon} i_{f_{\alpha}} .
$$


By (3.9), (3.10), (3.19), (3.30), [Z1, Proposition 2.2], and by proceeding similarly as in [BC1, (4.69)], after the conjugation by (3.4), the $G_{\sqrt{t \varepsilon}}$ rescaled operator of $t\left(D_{s, \varepsilon}^{\pi^{*} \mu \otimes F}(r)\right)^{2}-z \sqrt{t} D_{s, \varepsilon}^{\pi^{*} \mu \otimes F}(r)$ converges as $\varepsilon \rightarrow 0$ to

$$
\begin{aligned}
\mathcal{H}+\left(1+r^{2}\right)\left(C_{4 t}^{\mu}\right)^{2}-z & \left(\sqrt{t} D^{Z}+\frac{c(T)}{4 \sqrt{t}}+\sqrt{-1} r\left(\sqrt{t}\left(d^{Z *}-d^{Z}\right)+\frac{\widehat{c}(T)}{4 \sqrt{t}}\right)\right) \\
= & \mathcal{H}+\left(1+r^{2}\right)\left(C_{4 t}^{2}+R^{\mu}\right)-z 2 t \frac{\partial}{\partial t}\left(C_{4 t}+\sqrt{-1} r D_{4 t}\right),
\end{aligned}
$$

where

$$
\mathcal{H}=-\sum_{\alpha}\left(\nabla_{f_{\alpha}}+\frac{1}{4}\left\langle R_{b_{0}}^{T B} y, f_{\alpha}\right\rangle\right)^{2}-\frac{1}{4} \sum_{\alpha, \beta}\left\langle R_{b_{0}}^{T B} f_{\alpha}, f_{\beta}\right\rangle \widehat{c}\left(f_{\alpha}\right) \widehat{c}\left(f_{\beta}\right) .
$$

Finally, by the previous lemma, we see that the rescaled operator obtained from the conjugation by (3.4) of $\widehat{D}_{\varepsilon}^{\pi^{*} \mu \otimes F}$ converges to $D_{4 t}$ as $\varepsilon \rightarrow 0$. Proceeding as in [MZ] and noting $2 t \frac{\partial}{\partial t}=\frac{\partial}{\partial \sqrt{t}}$, we obtain the desired formula.

Proposition 3.6. We have the following uniform asymptotic expansion

$$
\operatorname{Tr}_{s}\left[\widehat{D}_{\varepsilon}^{\pi^{*} \mu \otimes F} D_{\varepsilon}^{\pi^{*} \mu \otimes F}(r) e^{-t\left(D_{\varepsilon}^{\pi^{*} \mu \otimes F}(r)\right)^{2}}\right]=c_{-k}(\varepsilon) t^{-k}+c_{-k+1}(\varepsilon) t^{-k+1}+\cdots,
$$

where $k=\frac{3}{2}$ if $n$ (dimension of the fiber) is odd and $k=1$ if $n$ is even. Similarly,

$$
\begin{gathered}
-\int_{B} L\left(T B, \nabla^{T B}\right) \operatorname{ch}\left(\mu, \nabla^{\mu}\right) \varphi \operatorname{Tr}_{s}\left[t^{-\frac{1}{2}} D_{4 t} \frac{\partial}{\partial \sqrt{t}}\left(C_{4 t}+\sqrt{-1} r D_{4 t}\right) e^{-\left(1+r^{2}\right) C_{4 t}^{2}}\right] \\
=c_{-k} t^{-k}+c_{-k+1} t^{-k+1}+\cdots .
\end{gathered}
$$

Moreover,

$$
c_{i / 2}(\varepsilon) \longrightarrow c_{i / 2} \quad \text { as } \varepsilon \rightarrow 0 .
$$

Proof. Using two auxiliary Grassmann variables $z_{1}, z_{2}$, we write

$$
\begin{aligned}
& \operatorname{Tr}_{s}\left[\widehat{D}_{\varepsilon}^{\pi^{*} \mu \otimes F} D_{\varepsilon}^{\pi^{*} \mu \otimes F}(r) e^{\left.-t\left(D_{\varepsilon}^{\pi^{*} \mu \otimes F}(r)\right)^{2}\right]}=\right. \\
&-t^{-2} \operatorname{Tr}_{s, z_{1}, z_{2}}\left[e^{-t\left(\left[D_{\varepsilon}^{\pi^{*} \mu \otimes F}(r)\right]^{2}-z_{1} D_{\varepsilon}^{\pi^{*} \mu \otimes F}(r)-z_{2} \widehat{D}_{\varepsilon}^{\pi^{*} \mu \otimes F}\right)}\right] .
\end{aligned}
$$

Applying the standard elliptic theory to the right hand side of (3.35), we derive an asymptotic expansion

$$
\begin{aligned}
\operatorname{Tr}_{s}\left[\widehat{D}_{\varepsilon}^{\pi^{*} \mu \otimes F} D_{\varepsilon}^{\pi^{*} \mu \otimes F}(r) e^{\left.-t\left(D_{\varepsilon}^{\pi^{*} \mu \otimes F}(r)\right)^{2}\right]=}\right. & c_{-m / 2-2}(\varepsilon) t^{-m / 2-2} \\
& +c_{-m / 2-1}(\varepsilon) t^{-m / 2-1}+\cdots .
\end{aligned}
$$


To prove the vanishing of the coefficients, we revert to one auxiliary Grassmann variable $z$ and rewrite

$$
\begin{aligned}
t^{\frac{3}{2}} \operatorname{Tr}_{s}\left[\widehat{D}_{\varepsilon}^{\pi^{*} \mu \otimes F} D_{\varepsilon}^{\pi^{*} \mu \otimes F}(r) e^{-t\left(D_{\varepsilon}^{\pi^{*} \mu \otimes F}(r)\right)^{2}}\right] & = \\
& -\operatorname{Tr}_{s, z}\left[t \widehat{D}_{\varepsilon}^{\pi^{*} \mu \otimes F} e^{-t\left(D_{\varepsilon}^{\pi^{*} \mu \otimes F}(r)\right)^{2}+z \sqrt{t} D_{\varepsilon}^{\pi^{*} \mu \otimes F}(r)}\right] .
\end{aligned}
$$

As usual, one fixes a point of $M$ and employs the normal coordinates $x$ around the point. Consider the Getzler rescaling $G_{\sqrt{t}}^{M}$ :

$$
x_{a} \rightarrow \sqrt{t} x_{a}, \quad \nabla_{e_{a}} \rightarrow \frac{1}{\sqrt{t}} \nabla_{e_{a}}, \quad c\left(e_{a}\right) \rightarrow \frac{1}{\sqrt{t}} e_{a} \wedge-\sqrt{t} i_{e_{a}} .
$$

By (3.27), (3.28), (3.29) and the same argument as in [BF], we can formulate a Lichnerowicz formula for $t\left(D_{\varepsilon}^{\pi^{*} \mu \otimes F}(r)\right)^{2}-z \sqrt{t} D_{\varepsilon}^{\pi^{*} \mu \otimes F}(r)$. The only singular term with respect to the Getzler rescaling $G_{\sqrt{t}}^{M}$ as $t \rightarrow 0$ appears in

$$
-t \varepsilon \sum_{a=1}^{p}\left(\nabla_{e_{a}}+\frac{z c\left(e_{a}\right)}{2 \sqrt{t \varepsilon}}\right)^{2}-t \sum_{a=p+1}^{m}\left(\nabla_{e_{a}}+\frac{z c\left(e_{a}\right)}{2 \sqrt{t}}\right)^{2} .
$$

This singular term can be easily eliminated by the exponential transform, namely conjugating by the exponential

$$
e^{\frac{z \sum_{a=1}^{m} x_{a} c\left(e_{a}\right)}{2 \sqrt{t}}} .
$$

Thus, after the exponential transform and then the Getzler rescaling $G_{\sqrt{t}}^{M}$, we find that $t\left(D_{\varepsilon}^{\pi^{*} \mu \otimes F}(r)\right)^{2}-z \sqrt{t} D_{\varepsilon}^{\pi^{*} \mu \otimes F}(r)$ converges as $t \rightarrow 0$ to

$$
\mathcal{H}(r, \varepsilon)-r^{2} \omega^{2}+z \sqrt{-1} r \sum_{a=p+1}^{m} \hat{c}\left(e_{a}\right) \partial_{a},
$$

where

$\mathcal{H}(r, \varepsilon)=-\varepsilon \sum_{a=1}^{p}\left(\partial_{a}+\frac{1}{4}\left\langle R_{p_{0}}^{T M} y, e_{a}\right\rangle\right)^{2}-\left(1+r^{2}\right) \sum_{a=p+1}^{m}\left(\partial_{a}+\frac{1}{4}\left\langle R_{p_{0}}^{T M} y, e_{a}\right\rangle\right)^{2}$.

On the other hand, after the exponential transform and then the Getzler rescaling $G_{\sqrt{t}}^{M}, t \widehat{D}_{\varepsilon}^{\pi^{*} \mu \otimes F}$ converges to

$$
-\frac{z}{2} \sum_{a=p+1}^{m} \hat{c}\left(e_{a}\right) e_{a} \wedge
$$

It follows that

$\lim _{t \rightarrow 0} t^{\frac{3}{2}} \operatorname{Tr}_{s}\left[\widehat{D}_{\varepsilon}^{\pi^{*} \mu \otimes F} D_{\varepsilon}^{\pi^{*} \mu \otimes F}(r) e^{-t\left(D_{\varepsilon}^{\pi^{*} \mu \otimes F}(r)\right)^{2}}\right]=-\int^{B} \sum_{a=p+1}^{m} \hat{c}\left(e_{a}\right) e_{a} \wedge e^{-\mathcal{H}(\varepsilon, r)+r^{2} \omega^{2}}$, 
where $\int^{B}$ denotes the Berezin integral (cf. [MZ, Page 604]).

Thus, we deduce that $c_{i}(\varepsilon)=0$ for $-n / 2-2 \leq i<-k$, with $k=\frac{3}{2}$ if $n$ is odd. On the other hand, if $n$ is even, the Berezin integral on the right hand side vanishes for parity reason, and thus $k=1$.

Now we show that the asymptotic expansion is uniform in $\varepsilon$. According to the discussion above, after the conjugation by (3.4), the $G_{\sqrt{\varepsilon}}$ rescaled operator of $\left(D_{s, \varepsilon}^{\pi^{*} \mu \otimes F}(r)\right)^{2}-z D_{s, \varepsilon}^{\pi^{*} \mu \otimes F}(r)$ converges as $\varepsilon \rightarrow 0$ to

$$
\mathcal{H}+\left(1+r^{2}\right)\left(C_{4 t}^{2}+R^{\mu}\right)-z\left(D^{Z}+\frac{c(T)}{4}+\sqrt{-1} r\left(\left(d^{Z *}-d^{Z}\right)+\frac{\widehat{c}(T)}{4}\right)\right) .
$$

Similarly, the $G_{\sqrt{\varepsilon}}$ rescaled operator of $\widehat{D}_{\varepsilon}^{\pi^{*} \mu \otimes F}$ converges to $D_{4}$. Since the asymptotic expansion of

$$
\operatorname{Tr}_{s}\left[\widehat{D}_{\varepsilon}^{\pi^{*} \mu \otimes F} D_{\varepsilon}^{\pi^{*} \mu \otimes F}(r) e^{-t\left(D_{\varepsilon}^{\pi^{*} \mu \otimes F}(r)\right)^{2}}\right]
$$

depends only on the local symbols of the rescaled operators of $\left(D_{s, \varepsilon}^{\pi^{*} \mu \otimes F}(r)\right)^{2}-$ $z D_{s, \varepsilon}^{\pi^{*} \mu \otimes F}(r)$ and $\widehat{D}_{\varepsilon}^{\pi^{*} \mu \otimes F}$, the coefficients $c_{i}(\varepsilon)$ of its asymptotic expansion converges uniformly to that of

$$
\operatorname{Tr}_{s}\left[D_{4} e^{-t\left(\mathcal{H}+\left(1+r^{2}\right)\left(C_{4 t}^{2}+R^{\mu}\right)-z\left(D^{Z}+\frac{c(T)}{4}+\sqrt{-1} r\left(d^{Z *}-d^{Z}+\frac{\widehat{c}(T)}{4}\right)\right)\right)}\right] .
$$

On the other hand, since

$$
\begin{gathered}
\operatorname{Tr}_{s}\left[D_{4 t} e^{-\left(\mathcal{H}+\left(1+r^{2}\right)\left(C_{4 t}^{\mu}\right)^{2}-z\left(\sqrt{t} D^{Z}+\frac{c(T)}{4 \sqrt{t}}+\sqrt{-1} r\left(\sqrt{t}\left(d^{Z *}-d^{Z}\right)+\frac{\widehat{c}(T)}{4 \sqrt{t}}\right)\right)\right)}\right]= \\
t^{-1 / 2} \operatorname{Tr}_{s}\left[D_{4} e^{-t\left(\mathcal{H}+\left(1+r^{2}\right)\left(C_{4 t}^{2}+R^{\mu}\right)-z\left(D^{Z}+\frac{c(T)}{4}+\sqrt{-1} r\left(d^{Z *}-d^{Z}+\frac{\widehat{c}(T)}{4}\right)\right)\right)}\right],
\end{gathered}
$$

we obtain (3.34) and also the convergence of asymptotic coefficients.

Corollary 3.7. The function $\delta_{\varepsilon}(F, r)(s)$ in (3.23) has a meromorphic continuation to the whole complex plane with $s=0$ a regular point.

Proof. The integral in (3.23) is convergent at $t=\infty$ since

$$
\operatorname{Tr}_{s}\left[\widehat{D}_{\varepsilon}^{\pi^{*} \mu \otimes F} D_{\varepsilon}^{\pi^{*} \mu \otimes F}(r) e^{-t\left(D_{\varepsilon}^{\pi^{*} \mu \otimes F}(r)\right)^{2}}\right]
$$

is exponentially decaying in $t$ as $t \rightarrow \infty$. On the other hand, it follows immediately from Proposition 3.6 that the integral is convergent at $t=0$ for $\Re s>k-1$. Moreover the standard method shows that $\delta_{\varepsilon}(F, r)(s)$ in (3.23) has a meromorphic continuation to the whole complex plane with simple poles at $s=k-1, k, \ldots$. However the possible simple pole at $s=0$ is canceled by that of $\Gamma(s)$. Hence $s=0$ is a regular point. From this discussion, we also derive 
the following formula

$$
\begin{gathered}
\delta_{\varepsilon}(F)(r)=\delta_{\varepsilon}(F, r)^{\prime}(0) \\
=-\frac{1}{2} \int_{0}^{1}\left(\operatorname{Tr}_{s}\left[\widehat{D}_{\varepsilon}^{\pi^{*} \mu \otimes F} D_{\varepsilon}^{\pi^{*} \mu \otimes F}(r) e^{-t\left(D_{\varepsilon}^{\pi^{*} \mu \otimes F}(r)\right)^{2}}\right]-c_{-k}(\varepsilon) t^{-k}\right) d t \\
-\frac{1}{2} \int_{1}^{+\infty} \operatorname{Tr}_{s}\left[\widehat{D}_{\varepsilon}^{\pi^{*} \mu \otimes F} D_{\varepsilon}^{\pi^{*} \mu \otimes F}(r) e^{-t\left(D_{\varepsilon}^{\pi^{*} \mu \otimes F}(r)\right)^{2}}\right] d t+C,
\end{gathered}
$$

where $C=c_{\frac{3}{2}}(\varepsilon)$ if $m$ is odd (and hence $k=\frac{3}{2}$ ) and $C=\frac{1}{2} \Gamma^{\prime}(1) c_{-1}(\varepsilon)$ if $m$ is even (and thus $k=-1$ ).

We now define our torsion form. As in the discussion above, we first define the corresponding zeta function

$$
\zeta_{\mathcal{T}}(s)=-\frac{1}{\Gamma(s)} \int_{0}^{\infty} t^{s-1} \varphi \operatorname{Tr}_{s}\left[N_{Z} D_{t}^{2} e^{\left(1+r^{2}\right) D_{t}^{2}}\right] d t
$$

From [BL, Theorem 3.21], $\operatorname{Tr}_{s}\left[N_{Z}\left(1+2 D_{t}^{2}\right) e^{\left(1+r^{2}\right) D_{t}^{2}}\right]$ has an asymptotic expansion as $t \rightarrow 0$ with no singular terms (i.e. no singular powers of $t$ ). By [BZ] and [DM], $\operatorname{Tr}_{s}\left[N_{Z} e^{\left(1+r^{2}\right) D_{t}^{2}}\right]$ has an asymptotic expansion as $t \rightarrow 0$ starting with the $t^{-l}$ term, with $l=0$ if $n$ is even and $l=\frac{1}{2}$ if $n$ is odd, compare MZ, (3.118)]. Hence $\operatorname{Tr}_{s}\left[N_{Z} D_{t}^{2} e^{\left(1+r^{2}\right) D_{t}^{2}}\right]$ has an asymptotic expansion as $t \rightarrow 0$ starting with the $t^{-l}$ term:

$$
\operatorname{Tr}_{s}\left[N_{Z} D_{t}^{2} e^{\left(1+r^{2}\right) D_{t}^{2}}\right] \sim A_{-l} t^{-l}+A_{-l+1} t^{-l+1}+\cdots .
$$

It follows that $\zeta_{\mathcal{T}}(s)$ has a meromorphic continuation to the whole complex plane with $s=0$ a regular point. Also, for later use, we note that

$$
\zeta_{\mathcal{T}}(0)=0
$$

when $n$ is odd; and

$$
\left\{\zeta_{\mathcal{T}}(0)\right\}^{[i]}=0,
$$

for $i>0$ when $n$ is even. Now we define our torsion form by

$$
\mathcal{T}_{r}=\zeta_{\mathcal{T}}^{\prime}(0)
$$

In fact, we have

$$
\begin{gathered}
\mathcal{T}_{r}=-\int_{0}^{1} \varphi\left(\operatorname{Tr}_{s}\left[N_{Z} D_{t}^{2} e^{\left(1+r^{2}\right) D_{t}^{2}}\right]-A_{-l} t^{-l}\right) \frac{d t}{t} \\
-\int_{1}^{\infty} \varphi \operatorname{Tr}_{s}\left[N_{Z} D_{t}^{2} e^{\left(1+r^{2}\right) D_{t}^{2}}\right] \frac{d t}{t}+C^{\prime},
\end{gathered}
$$

where $C^{\prime}=\varphi\left(A_{-l} \Gamma^{\prime}(1)\right)$ if $n$ is even, and $C^{\prime}=\varphi\left(2 A_{-l}\right)$ if $n$ is odd. 
We also introduce a variant of the torsion form. From (3.34), we have for $t \rightarrow 0$

$\operatorname{Tr}_{s}\left[t^{-\frac{1}{2}} D_{4 t} \frac{\partial}{\partial \sqrt{t}}\left(C_{4 t}+\sqrt{-1} r D_{4 t}\right) e^{\left(1+r^{2}\right) D_{4 t}^{2}}\right] \sim C_{-k} t^{-k}+C_{-k+1} t^{-k+1}+\cdots$.

Here $k$ is defined as in (3.34). Define

$$
\zeta_{\widetilde{\mathcal{T}}}(s)=-\frac{1}{\Gamma(s)} \int_{0}^{\infty} t^{s} \varphi \operatorname{Tr}_{s}\left[t^{-\frac{1}{2}} D_{4 t} \frac{\partial}{\partial \sqrt{t}}\left(C_{4 t}+\sqrt{-1} r D_{4 t}\right) e^{\left(1+r^{2}\right) D_{4 t}^{2}}\right] d t .
$$

As before, this zeta function has analytic continuation to the whole complex plane with $s=0$ a regular point. Therefore we can define

$$
\widetilde{\mathcal{T}}_{r}=\zeta_{\widetilde{\mathcal{T}}}^{\prime}(0)
$$

In fact, one has

$$
\begin{aligned}
\widetilde{\mathcal{T}}_{r}= & -\int_{0}^{1} \varphi\left(\operatorname{Tr}_{s}\left[t^{-\frac{1}{2}} D_{4 t} \frac{\partial}{\partial \sqrt{t}}\left(C_{4 t}+\sqrt{-1} r D_{4 t}\right) e^{\left(1+r^{2}\right) D_{4 t}^{2}}\right]-C_{-k} t^{-k}\right) d t \\
& -\int_{1}^{\infty} \varphi \operatorname{Tr}_{s}\left[t^{-\frac{1}{2}} D_{4 t} \frac{\partial}{\partial \sqrt{t}}\left(C_{4 t}+\sqrt{-1} r D_{4 t}\right) e^{\left(1+r^{2}\right) D_{4 t}^{2}}\right] d t+C^{\prime \prime}
\end{aligned}
$$

where $C^{\prime \prime}=2 \varphi C_{-\frac{3}{2}}$ if $m$ is odd (and hence $k=\frac{3}{2}$ ) and $C^{\prime \prime}=\Gamma^{\prime}(1) \varphi C_{-1}$ if $m$ is even (and thus $k=1$ ).

We are now ready for our main result.

Theorem 3.8. Under the assumption that the flat vector bundle $F$ over $M$ is fiberwise acyclic, the following identity holds,

$$
\lim _{\varepsilon \rightarrow 0} \delta_{\varepsilon}(F)(r)=\int_{B} L\left(T B, \nabla^{T B}\right) \operatorname{ch}\left(\mu, \nabla^{\mu}\right) \mathcal{T}_{r} .
$$

Proof. The proof follows the same line as in the proof of Theorem 2.5. Using Proposition 3.5, Proposition 3.6, (3.37) and (3.46), one deduce that

$$
\lim _{\varepsilon \rightarrow 0} \delta_{\varepsilon}(F)(r)=\frac{1}{2} \int_{B} L\left(T B, \nabla^{T B}\right) \operatorname{ch}\left(\mu, \nabla^{\mu}\right) \widetilde{\mathcal{T}}_{r}
$$

To derive the final result, we use

$$
2 u \frac{\partial}{\partial u} C_{u}=-\left[N_{Z}, D_{u}\right], \quad 2 u \frac{\partial}{\partial u} D_{u}=-\left[N_{Z}, C_{u}\right]
$$

(see [MZ, (3.81)]) to rearrange the right hand side of (3.25) as in (2.35). Namely, one has

$$
\begin{aligned}
-\operatorname{Tr}_{s}\left[t^{-\frac{1}{2}} D_{4 t} \frac{\partial}{\partial \sqrt{t}}\left(C_{4 t}+\sqrt{-1} r D_{4 t}\right) e^{\left(1+r^{2}\right) D_{4 t}^{2}}\right]= \\
-\quad \frac{2}{t} \operatorname{Tr}_{s}\left[N_{Z} D_{t}^{2} e^{\left(1+r^{2}\right) D_{t}^{2}}\right]+\frac{\sqrt{-1} r}{t} d \operatorname{Tr}_{s}\left[N_{Z} D_{t} e^{\left(1+r^{2}\right) D_{t}^{2}}\right] .
\end{aligned}
$$


It follows that $-2 A_{i}+\sqrt{-1} r d B_{i}=0$ if $i<-\frac{1}{2}$ and $-2 A_{i}+\sqrt{-1} r d B_{i}=C_{i-1}$ if $i \geq-\frac{1}{2}$, where $B_{i}$ is the coefficient of asymptotic expansion of $\operatorname{Tr}_{s}\left[N_{Z} D_{t} e^{\left(1+r^{2}\right) D_{t}^{2}}\right]$. Consequently, we obtain by using (3.42) and (3.46),

$$
\int_{B} L\left(T B, \nabla^{T B}\right) \operatorname{ch}\left(\mu, \nabla^{\mu}\right) \widetilde{\mathcal{T}}_{r}=2 \int_{B} L\left(T B, \nabla^{T B}\right) \operatorname{ch}\left(\mu, \nabla^{\mu}\right) \mathcal{T}_{r} .
$$

\subsection{Comparison with the Bismut-Lott torsion form}

Recall that the Bismut-Lott torsion form $\mathcal{T}\left(T^{H} M, g^{T Z}, h^{F}\right)$ is defined by

$$
\begin{aligned}
& \mathcal{T}\left(T^{H} M, g^{T Z}, h^{F}\right)=-\varphi \int_{0}^{+\infty}\left(\operatorname{Tr}_{s}\left[N_{Z}\left(1+2 D_{u}^{2}\right) e^{D_{u}^{2}}\right]\right. \\
& \left.-d\left(H\left(Z,\left.F\right|_{Z}\right)\right)-\left(\frac{n}{2} \chi(Z) \operatorname{rk}(F)-d\left(H\left(Z,\left.F\right|_{Z}\right)\right)\right)\left(1-\frac{u}{2}\right) e^{-u / 4}\right) \frac{d u}{2 u} .
\end{aligned}
$$

Now we note that the second and the third terms of the integrand, terms inserted in (3.49) to make the integral convergent, are degree 0 terms. Hence, for $i>0$,

$$
\left\{\mathcal{T}\left(T^{H} M, g^{T Z}, h^{F}\right)\right\}^{[i]}=-\int_{0}^{+\infty}\left\{\varphi \operatorname{Tr}_{s}\left[N_{Z}\left(1+2 D_{u}^{2}\right) e^{D_{u}^{2}}\right]\right\}^{[i]} \frac{d u}{2 u},
$$

where we denote by a superscript $[i]$ the $i$-form component of the corresponding form.

On the other hand, since

$$
\begin{gathered}
\left\{\operatorname{Tr}_{s}\left[N_{Z} D_{u}^{2} \exp \left(D_{u}^{2}\right)\right]\right\}^{[i]}=u^{-i / 2}\left\{\operatorname{Tr}_{s}\left[N_{Z} u D_{1}^{2} \exp \left(u D_{1}^{2}\right)\right]\right\}^{[i]}, \\
\left\{\operatorname{Tr}_{s}\left[N_{Z} \exp \left(D_{u}^{2}\right)\right]\right\}^{[i]}=u^{-i / 2}\left\{\operatorname{Tr}_{s}\left[N_{Z} \exp \left(u D_{1}^{2}\right)\right]\right\}^{[i]},
\end{gathered}
$$

one deduces that, for $\Re s$ sufficiently large,

$$
\begin{aligned}
\int_{0}^{\infty} u^{s}\left\{\operatorname{Tr}_{s}\left[N_{Z} D_{u}^{2} \exp \left(D_{u}^{2}\right)\right]\right\}^{[i]} \frac{d u}{u}=\int_{0}^{\infty} u^{s-\frac{i}{2}}\left\{\operatorname{Tr}_{s}\left[N_{Z} D_{1}^{2} \exp \left(u D_{1}^{2}\right)\right]\right\}^{[i]} d u \\
=\int_{0}^{\infty} u^{s-\frac{i}{2}} \frac{\partial}{\partial u}\left\{\operatorname{Tr}_{s}\left[N_{Z} \exp \left(u D_{1}^{2}\right)\right]\right\}^{[i]} d u \\
=\int_{0}^{\infty}(i-2 s) u^{s-\frac{i}{2}}\left\{\operatorname{Tr}_{s}\left[N_{Z} \exp \left(u D_{1}^{2}\right)\right]\right\}^{[i]} \frac{d u}{2 u}, \\
=\int_{0}^{\infty}(i-2 s) u^{s}\left\{\operatorname{Tr}_{s}\left[N_{Z} \exp \left(D_{u}^{2}\right)\right]\right\}^{[i]} \frac{d u}{2 u},
\end{aligned}
$$

Cf. [MZ, (3.140) and (3.141)]. We have used our assumption that $H^{*}\left(Z_{b},\left.F\right|_{Z_{b}}\right)=$ $\{0\}$.

Thus, for $i>0$,

$$
\begin{aligned}
\frac{1}{\Gamma(s)} \int_{0}^{+\infty} u^{s}\left\{\varphi \operatorname{Tr}_{s}\left[N_{Z}\left(1+2 D_{u}^{2}\right) e^{D_{u}^{2}}\right]\right\}^{[i]} \frac{d u}{2 u}= \\
\left(\frac{1}{i-2 s}+1\right) \frac{1}{\Gamma(s)} \int_{0}^{+\infty} u^{s-1}\left\{\varphi \operatorname{Tr}_{s}\left[N_{Z} D_{u}^{2} \exp \left(D_{u}^{2}\right)\right]\right\}^{[i]} d u
\end{aligned}
$$


Hence,

$$
\left\{\mathcal{T}\left(T^{H} M, g^{T Z}, h^{F}\right)\right\}^{[i]}=\left(1+r^{2}\right)^{1-\frac{i}{2}}\left\{\left(2+\ln \left(1+r^{2}\right)\right) \zeta_{\mathcal{T}}(0)+\frac{i+1}{i} \mathcal{T}_{r}\right\}^{[i]}
$$

as

$$
\left\{\zeta_{\mathcal{T}}(s)\right\}^{[i]}=-\left(1+r^{2}\right)^{-s+\frac{i}{2}-1} \frac{1}{\Gamma(s)} \int_{0}^{\infty} u^{s-1}\left\{\varphi \operatorname{Tr}_{s}\left[N_{Z} D_{u}^{2} \exp \left(D_{u}^{2}\right)\right]\right\}^{[i]} d u .
$$

In particular, using (3.39) and (3.40), we have

$$
\left\{\mathcal{T}_{r}\right\}^{[i]}=\frac{i}{i+1}\left(1+r^{2}\right)^{\frac{i}{2}-1}\left\{\mathcal{T}\left(T^{H} M, g^{T Z}, h^{F}\right)\right\}^{[i]}
$$

For the degree 0 component, one has

$$
\left\{\mathcal{T}_{r}\right\}^{[0]}=0
$$

This is a direct consequence of [BL, Theorem 3.29]. Thus, up to a scaling factor on each degree component, $\mathcal{T}_{r}$ captures the positive degree components of the Bismut-Lott real analytic torsion form.

\section{References}

[APS1] M. F. Atiyah, V. K. Patodi and I. M. Singer, Spectral asymmetry and Riemannian geometry I. Proc. Camb. Philos. Soc. 77 (1975), 43-69.

[APS2] M. F. Atiyah, V. K. Patodi and I. M. Singer, Spectral asymmetry and Riemannian geometry II. Proc. Camb. Philos. Soc. 78 (1975), 405-432.

[APS3] M. F. Atiyah, V. K. Patodi and I. M. Singer, Spectral asymmetry and Riemannian geometry III. Proc. Camb. Philos. Soc. 79 (1976), 71-99.

[BGV] N. Berline, E. Getzler and M. Vergne, Heat kernels and the Dirac operator, Grundl. Math. Wiss. 298, Springer, Berlin-Heidelberg-New York 1992.

[BerB] A. Berthomieu and J.-M. Bismut, Quillen metric and higher analytic torsion forms, J. Reine Angew. Math. 457 (1994), 85-184.

[B] J.-M. Bismut, The Atiyah-Singer index theorem for families of Dirac operators: two heat equation proofs. Invent. Math. 83 (1986), 91-151.

[BC1] J.-M. Bismut and J. Cheeger, $\eta$-invariants and their adiabatic limits. $J$. Amer. Math. Soc. 2 (1989), 33-70.

[BC2] Bismut, J.-M., Cheeger, J.: Families index for manifolds with boundary, superconnections and cones. I. J. Funct. Anal. 89, 313-363 (1990)

[BC3] Bismut, J.-M., Cheeger, J.: Families index for manifolds with boundary, superconnections and cones. II. J. Funct. Anal. 90, 306-354 (1990) 
[BF] J.-M. Bismut and D. S. Freed, The analysis of elliptic families, II. Comm. Math. Phys. 107 (1986), 103-163.

[BK] J.-M. Bismut and K. Köhler, Higher analytic torsion forms for direct images and anomaly formulas. J. Algebraic Geom. 1 (1992), no. 4, 647684.

[BL] J.-M. Bismut and J. Lott, Flat vector bundles, direct images and higher real analytic torsion. J. Amer. Math. Soc. 8 (1995), 291-363.

[BZ] J.-M. Bismut and W. Zhang, An extension of a theorem by Cheeger and Müller. Astérisque, tom. 205, Paris, 1992.

[C] Cheeger, J.: $\eta$-invariants, the adiabatic approximation and conical singularities. J. Diff. Geom. 26, 175-221 (1987)

[D] X. Dai, Adiabatic limits, non multiplicativity of signature and Leray spectral sequence. J. Amer. Math. Soc. 4 (1991), 265-321.

[DF] X. Dai and D. S. Freed, $\eta$ invariants and determinant lines. J. Math. Phys. 35 (1994), 5155-5194.

[DM] X. Dai and R. B. Melrose, Adiabatic limit, heat kernel and analytic torsion, preprint.

[DWW] W. Dwyer, M. Weiss and B. Williams, A parametrized index theorem for the algebraic K-theory Euler class. Acta Math. 190 (2003), 1-104.

[Fr] D. Freed, Reidemeister torsion, spectral sequences, and Brieskorn spheres. J. Reine Angew. Math. 429 (1992), 75-89.

[Fri] D. Fried, Fuchsian groups and Reidemeister torsion. The Selberg trace formula and related topics (Brunswick, Maine, 1984), Contemp. Math. 53 (1986), 141-163.

[LST] W. Lück, T. Schick, T. Thielmann, Torsion and fibrations. J. Reine Angew. Math. 498 (1998), 1-33.

[M] X. Ma, Functoriality of real analytic torsion forms. Israel J. Math. 131 (2002), 1-50.

[MP1] R. Melrose and P. Piazza, Families of Dirac operators, boundaries and the b-calculus. J. Differential Geom. 46 (1997), no. 1, 99-180.

[MP2] R. Melrose and P. Piazza, An index theorem for families of Dirac operators on odd-dimensional manifolds with boundary. J. Differential Geom. 46 (1997), no. 2, 287-334.

[MZ] X. Ma and W. Zhang, Eta-invariants, torsion forms and flat vector bundles. Math. Ann. 340 (2008), 569-624.

[Q] D. Quillen, Superconnections and the Chern character. Topology 24 (1986), 89-95. 
[RS] D. B. Ray and I. M. Singer, $R$-torsion and the Laplacian on Riemannian manifolds. Adv. Math. 7 (1971), 145-210.

[W] Witten, E.: Global gravitational anomalies. Comm. Math. Phys. 100, 197$229(1985)$

[Z1] W. Zhang, Sub-signature operators, $\eta$-invariants and a Riemann-Roch theorem for flat vector bundles. Chinese Ann. Math. 25B (2004), 7-36.

[Z2] W. Zhang, Sub-signature operators and a local index theorem for them. (in Chinese). Chinese Sci. Bull. 41 (1996), 294-295. 